\title{
A partial taxonomy of judgment aggregation rules and their properties *
}

\author{
Jérôme Lang Gabriella Pigozzi Marija Slavkovik \\ Leendert van der Torre Srdjan Vesic
}

April 11, 2018

\begin{abstract}
The literature on judgment aggregation is moving from studying impossibility results regarding aggregation rules towards studying specific judgment aggregation rules. Here we give a structured list of most rules that have been proposed and studied recently in the literature, together with various properties of such rules. We first focus on the majoritypreservation property, which generalizes Condorcet-consistency, and identify which of the rules satisfy it. We study the inclusion relationships that hold between the rules. Finally, we consider two forms of unanimity, monotonicity, homogeneity, and reinforcement, and we identify which of the rules satisfy these properties.
\end{abstract}

\section{Introduction}

Judgment aggregation studies the problems related to aggregating a finite set of yes-no individual judgments, cast on a collection of logically interrelated issues. Such a finite set of issues forms the agenda. It can be seen as a generalisation of preference aggregation [4.

Until a few years ago, the judgment aggregation literature had focused considerably more on studying impossibility theorems than on developing and investigating specific aggregation rules. This field development approach departs from the, admittedly much older, field of voting theory. Nevertheless, several recent and independent papers have started to explore the zoo of concrete judgment aggregation rules, beyond the well known premise-based and conclusionbased rules [8, 31]. While the premise- and conclusion-based rules can only be applied if there exists a prior labelling of the agenda issues as premises and conclusions, the following rules are defined for every agenda: quota-based rules [5], distance-based rules [30, 25, 14, 9], generalizations of Condorcet-consistent voting rules [28, 27, 21], and rules based on the maximisation of some scoring function $21,3,36$. Some of these rules obviously generalize well-known voting 
rules. However, a 'compendium' of existing judgment aggregation rules really does not exist at the moment, despite the several overview papers, chapters and even books that have been published in recent years [24, 18, 12, 1].

Our aim is threefold. First, as there is so far no compendium of judgment aggregation rules, we give one: we list most of the rules that have been proposed recently, in a structured way. This part of the paper does not give novel results, but serves as a partial survey. Second, we compare in a systematic way these rules in terms of inclusion relationships. Third, we consider a few key properties that generalize properties of voting rules (majority-preservation, unanimity, monotonicity, homogeneity and reinforcement) and identify those of the considered rules that satisfy them.

We follow earlier work in judgment aggregation 23 in using a constraintbased version of judgment aggregation to represent properties like transitivity of preferences. As it is common in voting theory, we consider irresolute rules (also called 'correspondences') rather than functions, that is, a rule outputs a non-empty set of collective judgments.

The outline of the paper is as follows. The general definitions are given in Section 2 In Section 3 we review the rules we study in the paper. Majority preservation is a key property of rules, as it generalizes Condorcet-consistency. We focus on majority-preservation in Section 4 and show which of the rules defined in Section 3 satisfy it. In Section 5 we address inclusion and noninclusion relationships between our rules. In Section 6 we study the rules from the point of view of unanimity, monotonicity, reinforcement and homogeneity. We summarize our contributions in Section 7

\section{Preliminaries}

Let $\mathcal{L}$ be a standard propositional language consisting of well-formed propositional logical formulas, including $T$ (tautology) and $\perp$ (contradiction), together with a standard notion of logical consistency. We denote atomic propositions by $p, q$ etc. and formulas from $\mathcal{L}$ by $\varphi, \alpha, \beta$ etc.

An agenda $\mathcal{A}$ is a finite set of propositions of the form $\left\{\varphi_{1}, \neg \varphi_{1}, \ldots, \varphi_{m}\right.$, $\left.\neg \varphi_{m}\right\}$, where for all $i, \varphi_{i} \in \mathcal{L}$ and $\varphi_{i}$ is neither a tautology nor a contradiction, and is a non-negated formula, (i.e., it is not of the form $\neg \alpha$ ). We refer to a pair $(\varphi, \neg \varphi)$ as an issue. The pre-agenda $[\mathcal{A}]$ associated with $\mathcal{A}$ is $[\mathcal{A}]=\left\{\varphi_{1}, \ldots, \varphi_{m}\right\}$. We slightly abuse notation and write $\varphi_{i}$ instead of $\neg \neg \varphi_{i}$ for $\varphi_{i} \in[\mathcal{A}]$.

An agenda is endowed with a notion of consistency which preserves logical consistency. Formally, $\mathcal{A}$ comes with a set of $(\mathcal{A}$-) consistent judgment sets; a $(\mathcal{A}$-)consistent judgment set is logically consistent, but the converse does not necessarily hold. Without loss of generality, the agenda's consistency notion is defined as logical consistency given some fixed formula: a set of formulas $S$ is consistent if $S \cup\{\gamma\}$ is logically consistent, where $\gamma$ is some exogenously fixed non-contradictory formula, which we call the integrity constraint. This is also the approach followed in [17] (albeit in the slightly different framework of binary 
aggregation, where agenda issues are atomic propositions) and in [13. A similar use of constraints is also done in belief merging theory [19, 16. When $\gamma$ is not specified, by default it is equal to $T$, in which case the notion of consistency associated with the agenda coincides with standard logical consistency.

A judgment on $\varphi \in[\mathcal{A}]$ is either $\varphi$ or $\neg \varphi$. A judgment set $J$ for $\mathcal{A}$ is a subset of $\mathcal{A}$. $J$ is complete if and only if for each $\varphi \in[\mathcal{A}]$, either $\varphi \in J$ or $\neg \varphi \in J$. A judgment set for $\mathcal{A}$ is rational if it is complete and consistent. Let $\mathcal{J}_{\mathcal{A}}$ be the set of all rational judgment sets for $\mathcal{A}$.

For every consistent $S \subseteq \mathcal{A}$, the set of rational extensions of $S$, i.e., $\{J \mid J \in$ $\mathcal{J}_{\mathcal{A}}$ and $\left.S \subseteq J\right\}$, is denoted as $\operatorname{ext}(S)$.

A $\mathcal{J}_{\mathcal{A}}$-profile, or simply a profile, is a finite sequence of rational individual judgment sets, i.e., $P=\left\langle J_{1}, \ldots, J_{n}\right\rangle$ for some $n$, where $J_{i}$ is the judgment set of voter $i$. We slightly abuse notation and write $J \in P$ when $J=J_{i}$ for some $i$, and we write $|P|$ to denote the number of judgment sets in $P$. We sometimes denote $P$ as $\left(J_{i}, J_{-i}\right)$, where $J_{-i}=\left\langle J_{j}, 1 \leq j \leq n, j \neq i\right\rangle$. We write $Q \subseteq P$ $\left(\right.$ read " $Q$ is a sub-profile of $P$ ) if $Q=\left\langle J_{j} \mid i \in I\right\rangle$ for some $I \subseteq\{1, \ldots, n\}$.

Given two rational judgment sets $J$ and $J^{\prime}$ we define the Hamming distance $d_{H}: d_{H}\left(J, J^{\prime}\right)$ as the number of issues on which $J$ and $J^{\prime}$ disagree. We also define the Hamming distance between two profiles $P=\left\langle J_{1}, \ldots, J_{n}\right\rangle$ and $P^{\prime}=$ $\left\langle J_{1}^{\prime}, \ldots, J_{n}^{\prime}\right\rangle$ as $D_{H}\left(P, P^{\prime}\right)=\sum_{i=1}^{n} d_{H}\left(J_{i}, J_{i}^{\prime}\right)$, and between a judgment set and a profile as $d_{H}(J, P)=\sum_{i=1}^{n} d_{H}\left(J, J_{i}\right)$.

We define $N(P, \varphi)$ as the number of all voters in $P$ whose judgment set contains $\varphi$, i.e., $N(P, \varphi)=\left|\left\{i \mid \varphi \in J_{i}, J_{i} \in P\right\}\right|$.

Example 1 Consider the pre-agenda $[\mathcal{A}]=\{p \wedge r, q, p \wedge q\}$. The corresponding agenda is $\mathcal{A}=\{p \wedge r, \neg(p \wedge r), q, \neg q, p \wedge q, \neg(p \wedge q)\}$, equipped with the consistency notion corresponding to $\gamma=(q \rightarrow r)$. The set of rational judgment sets is

$$
\mathcal{J}_{\mathcal{A}}=\left\{\begin{array}{lc}
\{\neg(p \wedge r), \neg q, \neg(p \wedge q)\}, & \{\neg(p \wedge r), q, \neg(p \wedge q)\}, \\
\{(p \wedge r), \neg q, \neg(p \wedge q)\}, & \{p \wedge r, q, p \wedge q\}
\end{array}\right\}
$$

Consider the profile $P=\left\langle J_{1}, J_{2}, J_{3}, J_{4}\right\rangle$ with $J_{1}=J_{2}=\{\neg(p \wedge r), q, \neg(p \wedge q)\}$, $J_{3}=\{p \wedge r, \neg q, \neg(p \wedge q)\}$ and $J_{4}=\{p \wedge r, q, p \wedge q\}$. For instance, $N(P, q)=3$.

Most often we will write profiles in a table, as in Table1, with the pre-agenda elements given in the topmost row and the voters' judgment sets in the leftmost column. If a judgment set contains $\varphi \in[\mathcal{A}]$ (respectively $\neg \varphi$ ), then we mark this with a "+" (respectively, "-") in the corresponding column. The constraint, if explicitly defined, will be given in the table caption.

The majoritarian judgment set associated with profile $P=\left\langle J_{1}, \ldots, J_{n}\right\rangle$ contains all the elements of the agenda that are supported by a strict majority of judgment sets in $P$, i.e., $m(P)=\left\{\varphi \in \mathcal{A} \mid N(P, \varphi)>\frac{n}{2}\right\}$. A profile $P$ is majority-consistent when $m(P)$ is a consistent subset of $\mathcal{A}$.

An (irresolute) judgment aggregation rule $F$ maps every profile $P$, defined on every agenda $\mathcal{A}$, to a nonempty set of rational judgment sets in $\mathcal{J}_{\mathcal{A}}$. When for all profiles $P, F(P)$ is a singleton, then $F$ is said to be resolute. Like in voting 
theory, resolute rules can be defined from irresolute ones by coupling them with a tie-breaking mechanism.

The preference pre-agendas associated with a set $C=\left\{x_{1}, \ldots, x_{q}\right\}$ of alternatives are defined by the set of atomic propositions $\left\{x_{i} P x_{j} \mid 1 \leq i<j \leq q\right\}$ (when $j>i, x_{j} P x_{i}$ is not an atomic proposition, but we write $x_{j} P x_{i}$ as a shorthand for $\left.\neg\left(x_{i} P x_{j}\right)\right)$ and one of these two consistency notions: transitivity, defined as consistency with

$$
\operatorname{Tr}=\bigwedge_{\substack{i, j, k \in\{1, \ldots, q\} \\ i \neq j \neq k}}\left(x_{i} P x_{j} \wedge x_{j} P x_{k} \rightarrow x_{i} P x_{k}\right)
$$

or existence of a nondominated alternative, defined as consistency with

$$
W=\bigvee_{i \in\{1, \ldots, q\}} \bigwedge_{j \neq i} x_{i} P x_{j}
$$

Finally, $\mathcal{A}_{C}^{T r}$ (respectively, $\mathcal{A}_{C}^{W}$ ) is defined by its pre-agenda $\left[\mathcal{A}_{C}^{T r}\right]=\left\{x_{i} P x_{j} \mid\right.$ $1 \leq i<j \leq q\}$ and the consistency notion corresponding to transitivity (respectively, with the existence of a nondominated alternative).

A preference profile over $C$ is a finite sequence of linear orders over $C$, which we denote by $V=\left\langle\succ_{1}, \ldots, \succ_{n}\right\rangle$. The majority graph $M(V)$ associated with $V$ is the directed graph whose vertices are elements of $C$ and containing edge $(x, y)$ if and only if a majority of voters in $V$ prefer $x$ to $y$; we denote by $n_{V}(x, y)$ the number of votes in $V$ that prefer $x$ to $y$. A social preference function maps every preference profile to a nonempty set of linear orders over $C$. A social choice function (or voting rule) maps every preference profile to a nonempty subset of $C$. With every judgment aggregation rule we can associate two social preference functions, whether we impose the transitivity constraint or the nondominated alternative constraint. From these two social preference functions we can derive two social choice functions by "collecting" the nondominated elements in each of the output preference relations. Sometimes, especially when $n$ is odd, these social preference functions or the social choice functions coincide with wellknown voting rules (we show several examples in Section 3).

\section{Judgment aggregation rules}

We now define five (overlapping) families of judgment aggregation rules. We use the following running example throughout the paper to illustrate the rules.

Example 2 Let $[\mathcal{A}]=\{p \wedge r, p \wedge s, q, p \wedge q, t\}$, and $P$ the 17-voter profile of Table 1. Consistency in $\mathcal{A}$ is logical consistency. As $m(P)=\{p \wedge r, p \wedge s, q, \neg(p \wedge q), t\}$ is an inconsistent judgment set, $P$ is not majority-consistent. 


\begin{tabular}{|c|c|c|c|c|c|}
\hline Voters & $p \wedge r$, & $p \wedge s$ & $q$, & $p \wedge q$, & $t\}$ \\
\hline$J_{1}-J_{6}$ & + & + & + & + & + \\
\hline$J_{7}-J_{10}$ & + & + & - & - & + \\
\hline$J_{11}-J_{17}$ & - & - & + & - & - \\
\hline $\mathrm{m}(\mathrm{P})$ & + & + & + & - & + \\
\hline
\end{tabular}

Table 1: Running example profile.

\subsection{Rules based on the majoritarian judgment set}

A judgment aggregation rule $F$ is based on the majoritarian judgment set when for every two $\mathcal{J}_{\mathcal{A}}$-profiles $P$ and $P^{\prime}$ such that $m(P)=m\left(P^{\prime}\right)$, we have $F(P)=$ $F\left(P^{\prime}\right)$. These rules can be viewed as the judgment aggregation counterparts of voting rules based on the pairwise majority graph, also known as C1 rules in Fishburn's classification (see, e.g., [2]).

Given a set of formulas $S \subseteq \mathcal{A}, S^{\prime} \subseteq S$ is a maximal consistent subset of $S$ if and only if $S^{\prime}$ is consistent and there exists no other consistent set $S^{\prime \prime}$ such that $S^{\prime} \subset S^{\prime \prime} \subseteq S$; and $S^{\prime} \subseteq S$ is a maxcard (for "maximal cardinality") consistent subset of $S$ if and only if $S^{\prime}$ is consistent and there exists no other consistent set $S^{\prime \prime} \subseteq S$ such that $\left|S^{\prime \prime}\right|>\left|S^{\prime}\right|$. The set of maximal (respectively, maxcard) consistent subsets of $S$ is denoted by $\max (S, \subseteq)(\operatorname{respectively,} \max (S,||)$.$) .$

Definition 1 (Maximal Condorcet and maxcard Condorcet rules) For

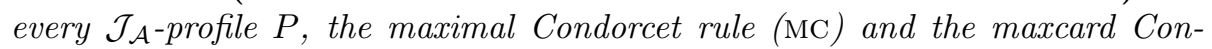
dorcet rule (MCC) are defined as follows:

$$
\begin{aligned}
\operatorname{MC}(P) & =\{\operatorname{ext}(S) \mid S \in \max (m(P), \subseteq)\}, \\
\operatorname{MCC}(P) & =\{\operatorname{ext}(S) \mid S \in \max (m(P),|.|)\} .
\end{aligned}
$$

Equivalently, $\operatorname{MCC}(P)=\underset{J \in \mathcal{J}_{\mathcal{A}}}{\operatorname{argmax}}|J \cap m(P)|$. Clearly, $\operatorname{MCC}(P) \subseteq \operatorname{MC}(P)$.

Example 3 For the profile $P$ of Example 2, the maximal consistent subsets of $m(P)$ are $\{p \wedge r, p \wedge s, q, t\},\{p \wedge r, p \wedge s, \neg(p \wedge q), t\}$ and $\{q, \neg(p \wedge q), t\}$; therefore

$$
\operatorname{MC}(P)=\left\{\begin{array}{ccccc}
\{p \wedge r, & p \wedge s, & q, & p \wedge q, & t\}, \\
\{p \wedge r, & p \wedge s, & \neg q, & \neg(p \wedge q), & t\}, \\
\{\neg(p \wedge r), & \neg(p \wedge s), & q, & \neg(p \wedge q), & t\}
\end{array}\right\}
$$

and

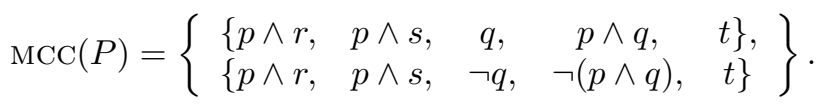

The output of the rule MC is called Condorcet admissible set by Nehring et al. 28]. The rule MCC is called Slater rule [28], and ENDPOINT $_{d_{H}}$ [25].

At least when $n$ is odd, it is easy to identify the voting rules obtained from MC and MCC. We give these results informally and without proof 1

\footnotetext{
${ }^{1}$ The proofs can be found in $[22$.
} 
- For MCC, the transitivity constraint leads to the social preference function that maps a profile to the set of all its Slater orders, i.e., the set of all linear orders $\succ$ over $C$ maximising the number of $(x, y)$ such that $x \succ y$ if and only if $(x, y) \in M(V)$, and that the corresponding voting rule (for $n$ odd) is the Slater rule, which maps a profile $V$ to the set of all alternatives that are dominating in some Slater order for $M(V)$. If we choose the $W$ constraint, then the corresponding voting rule (for $n$ odd) is the Copeland rule, which maps a profile to the set of alternatives $x$ maximising the number of outgoing edges from $x$ in $M(V)$.

- For MC, the transitivity constraint (for $n$ odd) leads to the top cycle rule, which maps a preference profile to the (unique) smallest subset $S$ of $C$ such that for every $x \in S$ and $y \in C \backslash S$, we have $(x, y) \in M(V) 2$ Finally, the choice of the $W$ constraint (for $n$ odd) leads to the voting rule that maps a profile to its Condorcet winner if and only if the profile has a Condorcet winner, and to the set of all alternatives otherwise.

\subsection{Rules based on the weighted majoritarian set}

The weighted majoritarian set associated with a profile $P$ is the function $N(P,$. which, we recall, maps each agenda issue to the number of judgment sets in $P$ that contain it. A judgment aggregation rule $F$ is based on the weighted majoritarian set when for every two $\mathcal{J}_{\mathcal{A}}$-profiles $P$ and $P^{\prime}$, if for every $\varphi \in \mathcal{A}$ we have $N(P, \varphi)=N\left(P^{\prime}, \varphi\right)$, then $F(P)=F\left(P^{\prime}\right)$. These rules can be viewed as the judgment aggregation counterparts of voting rules that are based on the weighted pairwise majority graph, also known as C2 rules in Fishburn's classification 2. Since $m(P)$ can be recovered from $N(P,$.$) , every rule based$ on the majoritarian judgment set is also based on the weighted majoritarian set.

Definition 2 (Median rule) For every $\mathcal{J}_{\mathcal{A}}$-profile $P$, the median rule (MED) is defined as follows:

$$
\operatorname{MED}(P)=\underset{J \in \mathcal{J}_{\mathcal{A}}}{\operatorname{argmax}} \sum_{\varphi \in J} N(P, \varphi) .
$$

This rule appears in many places under different names: Рвототуре [25], median rule [28], maximum weighted agenda rule [21], simple scoring rule [3] and distance-based procedure [14]. Variants of this rule have been defined by Konieczny and Pino-Pérez [19] and Pigozzi [30]. For completeness we give here the equivalent distance-based formulation of MED, although we consider more generally the family of distance-based rules in Section 3.4. For every $\mathcal{J}_{\mathcal{A}}$-profile $P$, the distance-based rule $F^{d_{H}, \Sigma}$ is defined as follows:

$$
F^{d_{H}, \Sigma}(P)=\underset{J \in \mathcal{J}_{\mathcal{A}}}{\operatorname{argmin}} \sum_{J_{i} \in P} d_{H}\left(J_{i}, J\right) .
$$

\footnotetext{
${ }^{2}$ This result has been independently proven (and stated in a stronger way) in [28].
} 
It is not difficult to establish that $F^{d_{H}, \Sigma}$ coincides with MED (see [21], and Proposition 1 in [3]). The social preference function obtained from MED and the choice of the transitivity constraint is the Kemeny social preference function, and the corresponding voting rule is the Kemeny rule.

Example 4 Consider the agenda and profile of Example 2. We obtain:

$\begin{array}{llll}N(P, p \wedge r)=10 & N(P, \neg(p \wedge r))=7 & N(P, p \wedge s)=10 & N(P, \neg(p \wedge s))=7 \\ N(P, q)=13 & N(P, \neg q)=4 & N(P, p \wedge q)=6 & N(P, \neg(p \wedge q))=11 \\ N(P, t)=10 & N(P, \neg t)=7 & & \end{array}$

As $\sum_{\varphi \in J} N(P, \varphi)$ reaches its maximum value (49) for $\{p \wedge r, p \wedge s, q, p \wedge q, t\}$, we have $\operatorname{MeD}(P)=\{\{p \wedge r, p \wedge s, q, p \wedge q, t\}\}$.

The following rule generalizes the ranked pairs voting rule 33. It proceeds by considering the elements $\varphi$ of the agenda in non-increasing order of $N(P, \varphi)$ and fixing each agenda issue value to the majoritarian value if it does not lead to an inconsistency.

Definition 3 (Ranked agenda rule) Let $\mathcal{A}=\left\{\psi_{1}, \ldots, \psi_{2 m}\right\}$. For every $\mathcal{J}_{\mathcal{A}}$-profile $P$, RA consists of those judgment sets $J \in \mathcal{J}_{\mathcal{A}}$ for which there exists a permutation $\left(\varphi_{1}, \varphi_{2}, \ldots, \varphi_{2 m}\right)$ of the propositions in $\mathcal{A}$ such that $N\left(P, \varphi_{1}\right) \geq$ $N\left(P, \varphi_{2}\right) \geq \cdots \geq N\left(P, \varphi_{2 m}\right)$ and $J$ is obtained by the following algorithmic procedure:

$$
\begin{aligned}
& S:=\emptyset \\
& \text { for } k=1, \ldots, 2 m \text { do } \\
& \quad \text { if } S \cup\left\{\varphi_{k}\right\} \text { is consistent then } S \leftarrow S \cup\left\{\varphi_{k}\right\} \\
& \quad \text { end if } \\
& \text { end for } \\
& J:=S
\end{aligned}
$$

In plain words, RA assigns iteratively a truth value to each proposition of the agenda, whenever it does not produce an inconsistency with propositions already assigned, following an order compatible with $N(P,$.$) . An equivalent$ non-procedural definition is the following: for every profile $P$, define $>_{P}^{R A}$ by: $J>{ }_{P}^{R A} J^{\prime}$ if there is an $\alpha \in \mathbb{N}$ such that

1. for all $\psi \in \mathcal{A}, N(P, \psi)>\alpha$ implies $\left[\psi \in J\right.$ if and only if $\left.\psi \in J^{\prime}\right]$, and

2. $J \cap\{\varphi \mid N(P, \varphi)=\alpha\} \supset J^{\prime} \cap\{\varphi \mid N(P, \varphi)=\alpha\}$.

Then $\operatorname{RA}(P)=\left\{J \in \mathcal{J}_{\mathcal{A}} \mid J\right.$ undominated in $\left.>_{P}^{R A}\right\} 3$

Example 5 Consider the profile of Example Q The highest value of $N(P, \varphi)$ is reached for $q$, therefore $q$ is fixed first. Then comes $\neg(p \wedge q)$, which is fixed as well. Then come $p \wedge r$ and $p \wedge s$, tied. We skip both because they would produce inconsistencies; then $t$ is fixed, and finally, $\neg(p \wedge r)$ and $\neg(p \wedge s)$. Thus,

$$
\operatorname{RA}(P)=\{\{q, \neg(p \wedge q), t, \neg(p \wedge r), \neg(p \wedge s)\}\} .
$$

\footnotetext{
${ }^{3}$ The proof-almost straightforward - can be found in 20].
} 
The Leximax rule [27, 15] is a refinement of RA. We repeat the definition of LEXIMAX here using our terminology.

Definition 4 Given an $n$-voter profile $P$ and a rational judgment set $J$, define $S_{k}(P)=\left\{\varphi \in \mathcal{A} \mid N(P, \varphi)=k, \frac{n}{2} \leq k \leq n\right\}$ and $s_{k}(J, P)=\left|S_{k}(P) \cap J\right|$. Given two rational judgment sets $J, J^{\prime}$, let $J>_{P}^{\text {leximax }} J^{\prime}$ if and only if there is a $k \in$ $\left\{\frac{n}{2}, \ldots, n\right\}$ such that $s_{k}(J, P)>s_{k}\left(J^{\prime}, P\right)$ and for all $i>k, s_{i}(J, P)=s_{i}\left(J^{\prime}, P\right)$. LEXIMAX $(P)$ is the set of all undominated rational judgment sets in with respect to $>>_{P}^{\text {leximax }}$.

For the profile $P$ of Example 22 $S_{13}(P)=\{q\}, S_{12}(P)=\emptyset, S_{11}(P)=$ $\{\neg(p \wedge q)\}, S_{10}(P)=\{p \wedge r, p \wedge s, t\}$. If $J=\{\neg(p \wedge r), \neg(p \wedge s), q, \neg(p \wedge q), t\}$ and $J^{\prime}=\{p \wedge r, p \wedge s, q, p \wedge q, t\}$, we have $s_{13}(J, P)=s_{13}\left(J^{\prime}, P\right)=1, s_{12}(J, P)=$ $s_{12}\left(J^{\prime}, P\right)=0, s_{11}(J, P)=0$ and $s_{11}\left(J^{\prime}, P\right)=1$, therefore $J>_{P}^{\text {leximax }} J^{\prime}$. (In fact, $J$ is the only $>_{P}^{\text {leximax }}$-undominated rational judgment set.)

It is easy to see that the social preference function (respectively, voting rule) associated with RA and the transitivity constraint is the ranked pairs social preference function (respectively, rule), which informally proceeds by iteratively fixing edges in the majority graph, whenever possible, considering all ordered pairs of alternatives $(x, y)$ in an order corresponding to non-increasing values of $n_{V}(x, y)$, and outputs the rankings obtained this way (respectively, the dominating elements in these rankings). However, the voting rule associated with RA and the $W$ constraint is the maximin rule, that maps a profile $V$ to the set of alternatives that maximise $\min _{y \in C \backslash\{x\}} n_{V}(x, y)$. The voting rules associated with LEXIMAX are refinements of ranked pairs and maximin.

\subsection{Rules based on elementary changes in profiles}

The next family of rules we consider contains rules that are based on minimal set of changes on a profile needed to render the profile majority-consistent. This family of judgment rules can be viewed as the judgment aggregation counterpart of voting rules that are rationalisable by some distance with respect to the Condorcet consensus class [11.

The first rule we consider is called the Young rule for judgment aggregation, by analogy with the Young voting rule, which outputs the candidate $c$ minimising the number of voters to remove from the profile so that $c$ becomes a weak Condorcet winner [34. The judgment aggregation generalization consists of removing a minimal number of voters so that the profile becomes majorityconsistent, or equivalently, to look for majority-consistent subprofiles of maximum cardinality.

Definition 5 (Young rule) For every $\mathcal{J}_{\mathcal{A}}$-profile $P$,

$$
\begin{aligned}
& \mathrm{Y}(P)=\{\operatorname{ext}(m(Q))|Q \in \quad \underset{Q \subseteq P,}{\operatorname{argmax}} \quad| Q \mid\} . \\
& m(Q) \text { is } \mathcal{A} \text {-consistent }
\end{aligned}
$$


Example 6 Once again we consider $\mathcal{A}$ and $P$ from Example 2. After noticing that removing three judgment sets from $\left\{J_{1}, \ldots, J_{6}\right\}$ restores majority-consistency, and removing less than three judgment sets does not, we obtain

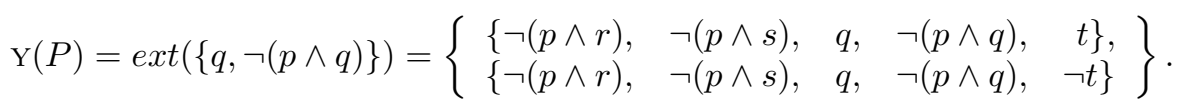

The voting rule associated with $\mathrm{Y}$ and the $W$ constraint is the Young voting rule 22 .

The next rule we define looks for a minimal number of individual judgment reversals in the profile so that $P$ becomes majority-consistent, where a judgment reversal is a change of truth value of one agenda element in one individual judgment set. This rule has been proposed first in Miller and Osherson 25] under the name $\mathrm{FULL}_{d}$. It bears a resemblance with the Dodgson voting rule, but does not exactly correspond to it when choosing either the $\operatorname{Tr}$ or the $W$ constraint.

Definition 6 (Minimal profile change rule) For $P \in \mathcal{J}_{\mathcal{A}}^{n}$, the MPC rule is defined as:

$$
\operatorname{MPC}(P)=\left\{\operatorname{ext}(m(Q)) \mid Q \in \underset{\substack{Q \in \mathcal{J}_{\mathcal{A}}^{n} \\ m(Q) \text { is } \mathcal{A} \text {-consistent }}}{\operatorname{argmin}} D_{H}(P, Q)\right\}
$$

Example 7 Consider the agenda $\mathcal{A}$ and profile $P$ of Example 2 Profile $P^{\prime}$ given in Table 2 is the closest majority-consistent profile to $P$ with $D_{H}\left(P, P^{\prime}\right)=$ 3 (the grey cell indicates the judgments reversed from $P$ ). We obtain $\operatorname{MPC}(P)=$ $\{\{p \wedge r, p \wedge s, q, p \wedge q, t\}\}$.

\begin{tabular}{r|ccccc} 
Voters & $\{p \wedge r$ & $p \wedge s$ & $q$ & $p \wedge q$ & $t\}$ \\
\hline$J_{1}-J_{6}$ & + & + & + & + & + \\
$J_{7}-J_{10}$ & + & + & - & - & + \\
$J_{11}-J_{14}$ & - & - & + & - & - \\
$J_{15}-J_{17}$ & - & - & + & + & - \\
\hline$m\left(P^{\prime}\right)$ & + & + & + & + & +
\end{tabular}

Table 2: The profile at minimal $D_{H}$ distance from the profile $P$ in Table 1

\subsection{Rules based on (pseudo-)distances}

For a given constrained agenda, a pseudo-distance $d$ on $\mathcal{J}_{\mathcal{A}}$ is a function that maps pairs of judgment sets to non-negative real numbers, and that satisfies, for all $J, J^{\prime} \in \mathcal{J}_{\mathcal{A}}, d\left(J, J^{\prime}\right)=d\left(J^{\prime}, J\right)$, and $d\left(J, J^{\prime}\right)=0$ if and only if $J=J^{\prime}$.

Two pseudo-distances we will use are the Hamming distance $d_{H}$, defined in Section 2, and the geodesic distanç4 on $\mathcal{J}_{\mathcal{A}}$, defined in [9] as follows. Given

\footnotetext{
${ }^{4}$ Our name; no name was given of this distance in $[9]$.
} 
three distinct rational judgment sets $J, J^{\prime}, J^{\prime \prime}$, we say that $J$ is between $J^{\prime}$ and $J^{\prime \prime}$ if $J^{\prime} \cap J^{\prime \prime} \subset J$. Let $G_{\mathcal{A}}$ be the graph whose set of vertices is the set of rational judgment sets $\mathcal{J}_{\mathcal{A}}$ and that contains an edge between $J^{\prime}$ and $J^{\prime \prime}$ if and only if there exists no $J \in \mathcal{J}_{\mathcal{A}}, J^{\prime} \neq J \neq J^{\prime \prime}$, between $J^{\prime}$ and $J^{\prime \prime}$. Finally, $d_{G}\left(J^{\prime}, J^{\prime \prime}\right)$ is defined as the length of the shortest path between $J^{\prime}$ and $J^{\prime \prime}$ in $G_{\mathcal{A}}$.

Definition 7 Let $d$ be a pseudo-distance on $\mathcal{J}_{\mathcal{A}}$ and $\star$ a commutative, associative and non-decreasing function on $\mathbb{R}^{+}$. The distance-based judgment aggregation rule $F^{d, \star}$ associated with $d$ and $\star$ is defined as

$$
F^{d, \star}(P)=\underset{J \in \mathcal{J}_{\mathcal{A}}}{\operatorname{argmin}} \star\left(d\left(J_{1}, J\right), \ldots, d\left(J_{n}, J\right)\right)
$$

In addition to $F^{d_{H}, \Sigma}$ we focus on two specific distance-based judgment aggregation rules: $F^{d_{G}, \Sigma}$, defined in [9], and $F^{d_{H}, \operatorname{MAx}}$, defined in [19, 21]. From now on, we will use the word 'distance' instead of 'pseudo-distance' although our rules can be defined more generally for pseudo-distances.

\subsection{Scoring rules}

Dietrich [3] defines a general class of scoring rules for judgment aggregation. Given a function $s: \mathcal{J}_{\mathcal{A}} \times \mathcal{A} \rightarrow \mathbb{R}^{+}$, the rule $F_{s}$ is defined as

$$
F_{s}(P)=\underset{J \in \mathcal{J}_{\mathcal{A}}}{\operatorname{argmax}} \sum_{\varphi \in J} \sum_{J_{i} \in P} s\left(J_{i}, \varphi\right) .
$$

The MED rule (3) is a scoring rule (and also a distance-based rule).

The reversal score function rev [3] is defined as:

$$
\operatorname{rev}(J, \varphi)=\min _{J^{\prime} \in \mathcal{J}_{\mathcal{A}}, \varphi \notin J^{\prime}} d_{H}\left(J, J^{\prime}\right) .
$$

The main motivation for introducing this rule is that the associated voting rule (with the transitivity constraint) is the Borda rule. Dietrich 3 defines four other scoring rules (entailment scoring, disjoint entailment scoring, minimal entailment scoring, and irreducible entailment scoring), two of which generalize the Borda rule as well. As he focuses on reversal scoring, we do as well, and leave the other four for further study beyond this paper.

Duddy et al. [10] introduce another interesting and intriguing scoring rule (defined only when the agenda satisfies a specific property); it generalizes not only the Borda rule, but also a well-behaved mean rule for finding collective dichotomies. We leave it for further study as well.

\section{Majority-preservation}

Intuitively, a judgment aggregation rule $F$ is majority-preserving if and only if $F$ returns only the extensions of the majoritarian judgment set whenever it is consistent. In case of ties, a majoritarian set can have more than one extension. 
For example, when we have agenda $\mathcal{A}=\{p, \neg p, q, \neg q\}$ and individual judgments $J_{1}=\{p, q\}$ and $J_{2}=\{p, \neg q\}$, then $m\left(\left\langle J_{1}, J_{2}\right\rangle\right)=\{p\}$, which can be extended into two complete collective judgment sets, namely $\{p, \neg q\}$ and $\{p, q\}$.

Definition 8 A judgment aggregation rule $F$ is majority-preserving if and only

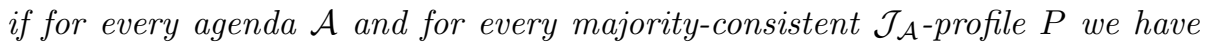
$F(P)=\operatorname{ext}(m(P))$. A rule $F$ is weakly majority-preserving if and only if for every agenda $\mathcal{A}$ and for every majority-consistent $\mathcal{J}_{\mathcal{A}}$-profile $P$ we have $F(P) \supseteq \operatorname{ext}(m(P))$.

Applied to the preference agenda with the transitivity constraint, majoritypreserving coincides with the requirement that a social welfare function should return the pairwise majority ordering whenever it is transitive; applied to the $W$ constraint, it coincides with the requirement that a social welfare function should return the pairwise majority ordering whenever it has a dominating element, i.e., whenever there is a Condorcet winner (which is slightly stronger than Condorcet-consistency).

Proposition 1 MC, MCC, MED, RA, LEXIMAX, Y and MPC are majority-preserving. $F^{d_{G}, \Sigma}$ and $F_{\text {rev }}$ are not even weakly majority-preserving.

Proof. Obvious cases are MC, MCC, MED, RA, LEXIMAx, y and MPC. For Frev, which coincides with the Borda rule for the preference agenda and the transitivity constraint, the result follows from the well-known fact that the Borda rule is not Condorcet-consistent. For $F^{d_{G}, \Sigma}$, consider the profile $P$ in Table 3

\begin{tabular}{r|cccccc} 
Voters & $\{p$, & $q$, & $r$, & $p \leftrightarrow q$, & $p \leftrightarrow r$, & $q \leftrightarrow r\}$ \\
\hline$J_{1}, J_{2}$ & + & + & + & + & + & + \\
$J_{3}, J_{4}, J_{5}$ & - & + & + & - & - & + \\
$J_{6}, J_{7}$ & + & - & + & - & + & - \\
$J_{8}, J_{9}$ & + & + & - & + & - & - \\
$J_{10}, J_{11}$ & - & - & - & + & + & + \\
\hline$m(P)$ & + & + & + & + & + & +
\end{tabular}

Table 3: A profile showing $F^{d_{G}, \Sigma}$ is not majority-preserving.

There are eight rational judgment sets over $\mathcal{A}$, i.e., $\left|\mathcal{J}_{\mathcal{A}}\right|=8$. We check that for every $J, J^{\prime} \in \mathcal{J}_{\mathcal{A}}$, if $J \neq J^{\prime}$ then $d_{G}\left(J, J^{\prime}\right)=1$. Therefore, $\sum_{J_{i} \in P} d_{G}\left(J_{3}, J_{i}\right)=$ 8. Now, $\sum_{J_{i} \in P} d_{G}\left(J, J_{i}\right)=9$ for every $J \in\left\{J_{1}, J_{6}, J_{8}, J_{10}\right\}$ and $\sum_{J_{i} \in P} d_{G}\left(J, J_{i}\right)=11$ for every $J \in\left(\mathcal{J}_{\mathcal{A}} \backslash\left\{J_{1}, J_{3}, J_{6}, J_{8}, J_{10}\right\}\right)$. Therefore, $F^{d_{G}, \Sigma}(P)=\left\{J_{3}\right\}$ although $P$ is majority-consistent and $m(P)=J_{1}$.

Let us call a pseudo-distance non-degenerate when there exist $J, J^{\prime}, J^{\prime \prime}$ such that $d\left(J, J^{\prime \prime}\right)>\max \left(d\left(J, J^{\prime}\right), d\left(J^{\prime}, J^{\prime \prime}\right)\right)$. Note that $d_{H}$ is non-degenerate. 
Proposition 2 For every distance $d$, the rule $F^{d, \mathrm{MAX}}$ is not majority-preserving. If $d$ is non-degenerate then $F^{d, \operatorname{MAx}}$ is not weakly majority-preserving.

Proof. Let $J_{1}, J_{2}$ be two distinct judgment sets such that $d\left(J_{1}, J_{2}\right) \leq d\left(J, J^{\prime}\right)$ for all $J \neq J^{\prime}$. Let $P=\left\langle J_{1}, J_{1}, J_{2}\right\rangle$. $P$ is majority-consistent, with $m(P)=J_{1}$, and yet $F^{d, \operatorname{MAx}}(P)$ contains also $J_{2}$, therefore $F^{d, \operatorname{MAx}}$ is not majority-preserving. If moreover $d$ is non-degenerate, let $J_{1}, J_{2}, J_{3}$ be three judgment sets such that $d\left(J_{1}, J_{3}\right)>\max \left(d\left(J_{1}, J_{2}\right), d\left(J_{2}, J_{3}\right)\right)$. Let $P=\left\langle J_{1}, J_{2}, J_{3}, J_{3}, J_{3}\right\rangle . P$ is majorityconsistent, with $m(P)=J_{3}$, and yet $F^{d, \operatorname{MAx}}(P)=\left\{J_{2}\right\}$, therefore $F^{d, \operatorname{MAx}}$ is not weakly majority-preserving.

Corollary $1 F^{d_{H}, \operatorname{MAx}}$ is not weakly majority-preserving.

\section{Inclusion relationships between the rules}

We now establish the following (non)inclusion relationships between most of the rules introduced so far. As the case-by-case proof is long and not very interesting, we chose to have it in the Appendix.

Definition 9 Given two judgment aggregation rules $F_{1}$ and $F_{2}$, we denote:

- $F_{1} \subseteq F_{2}$ when $F_{1}(P) \subseteq F_{2}(P)$ holds for every agenda $\mathcal{A}$ and every $\mathcal{J}_{\mathcal{A}^{-}}$ profile $P$.

- $F_{1} \subset F_{2}$ when $F_{1} \subseteq F_{2}$ and $F_{1} \neq F_{2}$.

- $F_{1}$ inc $F_{2}$ when neither $F_{1} \subseteq F_{2}$ nor $F_{2} \subseteq F_{1}$.

Let $F_{1} \in\left\{F^{d_{G}, \Sigma}, F_{\mathrm{rev}}, F^{d_{H}, \operatorname{MAx}}\right\}$ and $F_{2}$ be majority-preserving. Note that $F_{1}$ is not weakly majority-preserving, and that the counterexamples given in Section 4 all have an odd $n$. If $n$ is odd (recall that $m(P)$ is then a complete judgment set) then there is a majority-consistent profile $P$ for which $m(P) \notin$ $F_{1}(P)$, and $F_{2}(P)=\{m(P)\}$. This implies that $F_{1}$ inc $F_{2}$. Therefore, we have an incomparability relationship between a rule in $\left\{F^{d_{G}, \Sigma}, F_{\mathrm{rev}}, F^{d_{H}, \operatorname{MAx}}\right\}$ and a rule in $\{$ MC, MCC, MED, RA, LEXIMAX, Y, MPC .

Proposition 3 The inclusion and incomparability relations among the majoritypreserving rules, and among the non majority-preserving rules, are represented on Tables 4 and 5; $a \supset$ sign for row $F_{1}$ and column $F_{2}$ means that $F_{1} \supset F_{2}$, and an inc sign, that $F_{1}$ inc $F_{2}$.

\section{Unanimity, monotonicity, homogeneity, rein- forcement}

In preference aggregation, there are three classes of properties [37: those that are satisfied by most common rules (such as neutrality or anonymity); those 


\begin{tabular}{|r||c|c|c|c|c|c|}
\hline & MCC & MED & RA & LEXIMAX & Y & MPC \\
\hline MC & $\supset$ & $\supset$ & $\supset$ & $\supset$ & inc & inc \\
\hline MCC & & inc & inc & inc & inc & inc \\
\hline MED & & inc & inc & inc & inc \\
\hline RA & \multicolumn{7}{r|}{} & $\supset$ & inc & inc \\
\hline LEXIMAX & \multicolumn{7}{l}{} & inc & inc \\
\hline Y & & & inc \\
\hline
\end{tabular}

Table 4: (Non)inclusion relationships between the majority-preserving rules.

\begin{tabular}{|r||c|c|}
\hline & $F^{d_{G}, \Sigma}$ & $F_{\text {rev }}$ \\
\hline$F^{d_{H}, \mathrm{MAX}}$ & inc & inc \\
\hline$F^{d_{G}, \Sigma}$ & & inc \\
\hline
\end{tabular}

Table 5: (Non)inclusion relationships between the other rules.

that are very hard to satisfy, and whose satisfaction, under mild additional condition, implies impossibility results; and finally, those that are satisfied by a significant number of rules and violated by another significant number of rules. Similarly, in judgment aggregation, weak properties such as anonymity are satisfied by all our rules, while strong properties such as independence are violated by all our rules. We have already studied an "intermediate" property: majority-preservation. Here we consider four more: unanimity, monotonicity, homogeneity and reinforcement.

\subsection{Unanimity}

Unanimity has been defined for resolute rules by Dietrich and List [7]: $F$ is said to satisfy unanimity when for every $\mathcal{J}_{\mathcal{A}}$-profile $P=\left\langle J_{1}, \ldots, J_{n}\right\rangle$ and every $\varphi \in \mathcal{A}$, if $\varphi \in J_{i}$ for all $i \leq n$, then $\varphi \in F(P) 5$ We first generalise unanimity to irresolute rules, which gives us a weak and a strong version of unanimity.

Definition 10 (Weak and strong unanimity) Given $\varphi \in \mathcal{A}$, the $\mathcal{J}_{\mathcal{A}}$-profile $P$ is said to be $\varphi$-unanimous when $\varphi \in J_{i}$ for every $J_{i} \in P$.

- F satisfies weak unanimity when for every $\varphi$-unanimous profile $P$, there is a $J \in F(P)$ such that $\varphi \in J$.

- F satisfies strong unanimity when for every $\varphi$-unanimous profile $P$, for all $J \in F(P)$ we have $\varphi \in J$.

Proposition $4 \mathrm{MCC}, \mathrm{MED}, F^{d_{H}, \mathrm{MAx}}, \mathrm{MPC}, F^{d_{G}, \Sigma}$ and $F_{r e v}$ do not even satisfy weak unanimity.

Proof.

\footnotetext{
${ }^{5}$ A weaker unanimity property has been defined by List and Puppe [24], for resolute rules as well: $F(P)=J$ whenever all the voters in $P$ have the judgment set $J$.
} 
1. MCC, MED and MPC: The proof that MED does not satisfy weak unanimity can be found in 31. For MCC and MPC consider the profile $P$ from Table 6. $\operatorname{MCC}(P)$, and $\operatorname{MPC}(P)$ coincide: $\operatorname{MCC}(P)=\operatorname{MPC}(P)=\{\neg p, p \rightarrow$

\begin{tabular}{r|cccccccccc} 
Voters & $p$ & $p \rightarrow q \vee r$ & $q$ & $r$ & $p \rightarrow s \vee t$ & $s$ & $t$ & $p \rightarrow u \vee v$ & $u$ & $v$ \\
\hline$J_{1}$ & + & + & + & - & + & + & - & + & + & - \\
$J_{2}$ & + & + & - & + & + & - & + & + & - & + \\
$J_{3}$ & + & - & - & - & - & - & - & - & - & - \\
\hline $\mathrm{m}(\mathrm{P})$ & + & + & - & - & + & - & - & + & - & -
\end{tabular}

Table 6: A profile showing that $\operatorname{MCC}(P)$ and $\operatorname{MPC}(P)$ do not satisfy weak unanimity.

$(q \vee r), \neg q, \neg r, p \rightarrow(s \vee t), \neg s, \neg t, p \rightarrow(u \vee v), \neg u, \neg v\} . \operatorname{MPC}(P)$ is obtained by reversing two $p$ judgments in either two of the three judgment sets of the profile.

2. $F^{d_{H}, \operatorname{MAx}}$ : Let $[\mathcal{A}]=\{p, q, r, s, \alpha\}$, where $\alpha=(p \wedge q \wedge r \wedge s) \vee(\neg p \wedge$ $\neg q \wedge \neg r \wedge \neg s)$, and $P=\left\langle J_{1}, J_{2}\right\rangle$ where $J_{1}=\{p, q, r, s, \alpha\}$ and $J_{2}=$ $\{\neg p, \neg q, \neg r, \neg s, \alpha\} . F^{d_{H}, \operatorname{MAx}}(P)$ selects all $J \in \mathcal{J}_{\mathcal{A}}$ for which $\max \left(d_{H}\left(J, J_{1}\right), d_{H}\left(J, J_{2}\right)\right)=$ 3. For all such $J$ it holds that $\alpha \notin J$, although there is unanimity on $\alpha$.

3. $F^{d_{G}, \Sigma}$. Let $[\mathcal{A}]=\left\{p_{1}, p_{2}, \ldots, p_{13}\right\}$ with $\mathcal{J}_{\mathcal{A}}$ as given in Table 7 .

\begin{tabular}{r|ccccccccccccc} 
Sets & $\left\{p_{1}\right.$, & $p_{2}$, & $p_{3}$, & $p_{4}$, & $p_{5}$, & $p_{6}$, & $p_{7}$, & $p_{8}$, & $p_{9}$, & $p_{10}$, & $p_{11}$, & $p_{12}$ & $\left.p_{13}\right\}$ \\
\hline$J^{1}$ & + & - & - & + & - & - & + & - & - & + & - & - & + \\
$J^{2}$ & + & + & - & + & + & - & + & + & - & + & + & - & + \\
$J^{3}$ & - & + & - & - & + & - & - & + & - & - & + & - & + \\
$J^{4}$ & - & + & + & - & + & + & - & + & + & - & + & + & + \\
$J^{5}$ & - & - & + & - & - & + & - & - & + & - & - & + & + \\
$J^{6}$ & + & - & + & + & - & + & + & - & + & + & - & + & + \\
$J^{7}$ & - & - & - & - & - & - & - & - & - & - & - & - & -
\end{tabular}

Table 7: The $\mathcal{J}_{\mathcal{A}}$ for the example demonstrating that $F^{d_{G}, \Sigma}$ does not satisfy weak unanimity.

Consider the profile $P=\left\langle J_{1}, J_{2}, J_{3}\right\rangle$, where $J_{1}=J^{1}, J_{2}=J^{3}$, and $J_{3}=$ $J^{5}$. We have that $F^{d_{G}, \Sigma}(P)=\left\{J^{7}\right\}$, although there is unanimity on $p_{13}$. The $d_{G}$ distances between each set in $\mathcal{J}$ are given in Table 8 ,

4. $F_{\text {rev }}$. Consider a pre-agenda of $[\mathcal{A}]=\left\{p_{1}, p_{2}, \ldots, p_{13}\right\}$ with $\mathcal{J}_{\mathcal{A}}$ as given in Table 9. Consider the profile $P=\left\langle J_{1}, J_{2}, J_{3}\right\rangle$ where $J_{1}=J, J_{2}=J^{\prime}$, and $J_{3}=J^{\prime \prime}$. For $1 \leq i \leq 3$ and $1 \leq j \leq 13$, we have that $\operatorname{rev}\left(J_{i}, p_{j}\right)=5$ and $\operatorname{rev}\left(J_{i}, \neg p_{j}\right)=8$. We have that $F_{\mathrm{rev}}(P)=\left\{J^{4}\right\}$ since the score of $J^{4}$ for $P$ is 192, while the score of each of the profile judgment sets to $P$ is 163. 


\begin{tabular}{c|ccccccc}
$d_{G}(.,)$. & $J^{1}$ & $J^{2}$ & $J^{3}$ & $J^{4}$ & $J^{5}$ & $J^{6}$ & $J^{7}$ \\
\hline$J^{1}$ & 0 & 1 & 2 & 3 & 2 & 1 & 1 \\
$J^{2}$ & 1 & 0 & 1 & 2 & 3 & 2 & 2 \\
$J^{3}$ & 2 & 1 & 0 & 1 & 2 & 3 & 1 \\
$J^{4}$ & 3 & 2 & 1 & 0 & 1 & 2 & 2 \\
$J^{5}$ & 2 & 3 & 2 & 1 & 0 & 1 & 1 \\
$J^{6}$ & 1 & 2 & 3 & 2 & 1 & 0 & 2 \\
$J^{7}$ & 1 & 2 & 1 & 2 & 1 & 2 & 0
\end{tabular}

Table 8: The $d_{G}$ distances among the sets in $\mathcal{J}_{\mathcal{A}}$ from Table 7

\begin{tabular}{r|ccccccccccccc} 
Sets & $\left\{p_{1}\right.$, & $p_{2}$, & $p_{3}$, & $p_{4}$, & $p_{5}$, & $p_{6}$, & $p_{7}$, & $p_{8}$, & $p_{9}$, & $p_{10}$, & $p_{11}$, & $p_{12}$ & $\left.p_{13}\right\}$ \\
\hline$J^{1}$ & + & - & - & + & - & - & + & - & - & + & - & - & + \\
$J^{2}$ & - & + & - & - & + & - & - & + & - & - & + & - & + \\
$J^{3}$ & - & - & + & - & - & + & - & - & + & - & - & + & + \\
$J^{4}$ & - & - & - & - & - & - & - & - & - & - & - & - & -
\end{tabular}

Table 9: The $\mathcal{J}_{\mathcal{A}}$ for the example demonstrating that $F_{\text {rev }}$ does not satisfy weak unanimity.

This failure of $F_{\text {rev }}$ to satisfy unanimity is a surprising result, because the Borda social preference function (which ranks alternatives in a way consistent with their Borda scores) satisfies Pareto-efficiency.

Proposition 5 MC satisfies weak unanimity but not strong unanimity.

Proof. Let $P$ be a $\varphi$-unanimous $\mathcal{J}_{\mathcal{A}}$-profile for some $\varphi \in \mathcal{A}$. Note that for each $\psi \in m(P)$, there exists at least one $S \in \max (m(P), \subseteq)$ such that $\psi \in S$. Consequently there exists a judgment set in $\operatorname{MC}(P)$ that contains $\varphi$.

As a counter-example for MC satisfying strong unanimity, consider the profile $P$ of Table 6 MC does not satisfy strong unanimity since there exists $J \in$ $\operatorname{MC}(P)$ such that $\neg p \in J$. Namely, $\{\neg p, \neg(p \rightarrow(q \vee r)), \neg q, \neg r, \neg(p \rightarrow(s \vee$ $t)), \neg s, \neg t, \neg(p \rightarrow(u \vee v)), \neg u, \neg v\} \in \operatorname{MC}(P) 6$.

Proposition 6 RA, LEXIMAX and Y satisfy strong unanimity.

Proof. For RA and leximax: Let $P$ be a profile and $S \subseteq \mathcal{A}$ be the subset of the agenda consisting of all $\varphi \in \mathcal{A}$ for which $P$ is $\varphi$-unanimous. Because individual judgment sets are consistent, the conjunction of all elements of $S$ is consistent.

\footnotetext{
${ }^{6} \mathrm{MC}$ failing to satisfy strong unanimity is also a consequence of Theorem 2.2 in 29, which can be reformulated as: MC satisfies strong unanimity if and only if $\mathcal{A}$ does not contain a minimal inconsistent subset of size 3 or more.
} 
Now, when computing RA $(P)$, the elements of $S$ are considered first, and whatever the order in which they are considered, they are included in the resulting judgment set because no inconsistency arises. Therefore, for all $\varphi \in S$ and all $J \in \operatorname{RA}(P)$, we have $\varphi \in J$. Since Leximax $\subset$ RA, LEXimax satisfies strong unanimity as well.

For Y: If $\varphi$ is unanimously accepted in $P$, it is also unanimously accepted in every majority-consistent subprofile of $P$ and in its majoritarian judgment set.

\subsection{Monotonicity}

In voting, monotonicity states that when the position of the winning alternative improves in some vote ceteris paribus, then this alternative remains the winner. We define below a generalisation of this property for (irresolute) judgment aggregation rules. It is a generalization of the monotonicity property defined by Dietrich and List [6] for resolute rules.

\section{Definition 11 (Monotonicity)}

Let $P, P^{\prime} \in \mathcal{J}_{\mathcal{A}}^{n}$ be two profiles, and $\varphi \in \mathcal{A} . P^{\prime}$ is a $\varphi$-improvement of $P$ when (a) $P=\left(J_{i}, J_{-i}\right)$, (b) $P^{\prime}=\left(J_{i}^{\prime}, J_{-i}\right)$, (c) $\neg \varphi \in J_{i}$, (d) $\varphi \in J_{i}^{\prime}$, and (e) for all $\psi \in \mathcal{A}, \psi \notin\{\varphi, \neg \varphi\}, \psi \in J_{i}$ if and only if $\psi \in J_{i}^{\prime}$. (Note that the definition implies that $J_{i}^{\prime}$ is consistent, otherwise $P^{\prime}$ would not be a well-defined profile.) A judgment aggregation rule $F$ is monotonic, when for every $P \in \mathcal{J}_{\mathcal{A}}^{n}$ and its $\varphi$-improvement $P^{\prime} \in \mathcal{J}_{\mathcal{A}}^{n}$, for any $\varphi \in \mathcal{A}$, it holds that: if $\varphi \in J$ for every $J \in F(P)$, then $\varphi \in J^{\prime}$ for every $J^{\prime} \in F\left(P^{\prime}\right)$.

Note that not every profile has a $\varphi$-improvement for a given $\varphi \in \mathcal{A}$ : in Example1 $\{p \wedge r, \neg q, \neg(p \wedge q)\}$ is a $p \wedge r$-improvement of $\{\neg(p \wedge r), \neg q, \neg(p \wedge q)\}$, but $\{p \wedge r, q, p \wedge q\}$ has no $\psi$-improvement $\nabla$ for every $\psi \in\{\neg(p \wedge r), \neg q, \neg(p \wedge q)\}$.

In all the proofs of this section, $\mathcal{A}$ is an agenda, $\varphi$ an element of $\mathcal{A}, P=$ $\left(J_{i}, P_{-i}\right)$ a $\mathcal{J}_{\mathcal{A}}$-profile, and $P^{\prime}=\left(J_{i}^{\prime}, P_{-i}\right)$ a $\varphi$-improvement of $P$.

We start by proving the following lemmas, which will be useful for rules based on the majoritarian judgment set.

Lemma 1 Let $P$ a profile and $P^{\prime}$ a $\varphi$-improvement of $P$. Then one of the following three statements is true:

1. $m\left(P^{\prime}\right)=m(P)$;

2. $\neg \varphi \in m(P)$ and $m\left(P^{\prime}\right)=m(P) \backslash\{\neg \varphi\}$;

3. $\varphi \notin m(P)$ and $m\left(P^{\prime}\right)=(m(P) \backslash\{\neg \varphi\}) \cup\{\varphi\}$.

\footnotetext{
${ }^{7}$ Recall that there is a constraint $\gamma=q \rightarrow r$ for the agenda in this example.
} 
Proof. If $P^{\prime}$ is a $\varphi$-improvement of $P$ then $N\left(P^{\prime}, \varphi\right)=N(P, \varphi)+1$ and for all $\psi \in \mathcal{A} \backslash\{\varphi, \neg \varphi\}, N\left(P^{\prime}, \psi\right)=N(P, \psi)$. Table 10 represents the different possible cases concerning the presence or not of $\varphi$ and $\neg \varphi$ in $m(P)$ and $m\left(P^{\prime}\right)$, and which of the three statements 1, 2 and 3 holds. Obviously, the columns $=\frac{n}{2}-1$ and $=\frac{n}{2}$ are relevant only when $n$ is even, and the column $=\frac{(n-1)}{2}$ is relevant only when $n$ is odd. In all cases, one of 1,2 and 3 holds.

\begin{tabular}{c|c|c|c|c|c}
$N(P, \varphi)$ & $<\frac{n}{2}-1$ & $=\frac{n}{2}-1$ & $=\frac{n-1}{2}$ & $=\frac{n}{2}$ & $>\frac{n}{2}$ \\
\hline$m(P) \cap\{\varphi, \neg \varphi\}$ & $\{\neg \varphi\}$ & $\{\neg \varphi\}$ & $\{\neg \varphi\}$ & $\emptyset$ & $\{\varphi\}$ \\
\hline$N\left(P^{\prime}, \varphi\right)$ & $<\frac{n}{2}$ & $=\frac{n}{2}$ & $=\frac{n+1}{2}$ & $=\frac{n}{2}+1$ & $>\frac{n}{2}$ \\
\hline$m\left(P^{\prime}\right) \cap\{\varphi, \neg \varphi\}$ & $\{\neg \varphi\}$ & $\emptyset$ & $\{\varphi\}$ & $\{\varphi\}$ & $\{\varphi\}$ \\
\hline statement holding & 1 & 2 & 3 & 3 & 1
\end{tabular}

Table 10: Different possible cases concerning the presence or not of $\varphi$ and $\neg \varphi$ in $m(P)$ and $m\left(P^{\prime}\right)$

Lemma 2 Given a consistent judgment set $J$, if every rational extension of $J$ contains $\varphi$, then (a) $J$ does not contain $\neg \varphi$ and (b) $\operatorname{ext}(J \cup\{\varphi\})=\operatorname{ext}(J)$.

Proof. Assume that every rational extension of $J$ contains $\varphi$. A complete extension of $J$ containing $\neg \varphi$ would contain both $\varphi$ and $\neg \varphi$ and would not be consistent, hence (a) holds. For (b): because every rational extension of $J$ contains $\varphi$, every rational extension of $J$ is also a rational extension of $J \cup\{\varphi\}$, and obviously a rational extension of $J \cup\{\varphi\}$ is also a rational extension of $J \square$

Lemma 3 connects the monotonicity property with orders $\succ_{P}$ that rank the judgment sets in $\mathcal{J}_{\mathcal{A}}$ with respect to given profile $P \in \mathcal{J}_{\mathcal{A}}^{n}$. We consider the rules $F$ which select as collective judgments for $P \in \mathcal{J}_{\mathcal{A}}^{n}$ the undominated $J \in \mathcal{J}_{\mathcal{A}}$ based on some order $\succ_{P}$. This is condition (c) in Lemma 3. Such rules satisfy monotonicity when the order $\succ_{P}$ satisfies two properties. Intuitively, conditions (a) and (b) of Lemma 3, say that when going from $\succ_{P}$ to $\succ_{P^{\prime}}$, judgment sets containing $\varphi$ (respectively $\neg \varphi$ ) can only move "upward" (respectively "downward") in the preference relation.

Lemma 3 Let $F$ be a judgment aggregation rule such that there is a family of partial orders $\left(\succ_{P}\right)_{P \in \mathcal{J}_{\mathcal{A}}^{n}}$ over $\mathcal{J}_{\mathcal{A}}$ such that for every profile $P$, every $\varphi$ improvement $P^{\prime}$ of $P$, and all $J, J^{\prime} \in \mathcal{J}_{\mathcal{A}}$,

(a) if $\left[\varphi \in J\right.$ if and only if $\left.\varphi \in J^{\prime}\right]$, then $\left[J \succ_{P} J^{\prime}\right.$ implies $\left.J \succ_{P^{\prime}} J^{\prime}\right]$;

(b) if $\left[\varphi \in J\right.$ and $\left.\neg \varphi \in J^{\prime}\right]$, then $\left[J \succ_{P} J^{\prime}\right.$ implies $\left.J \succ_{P^{\prime}} J^{\prime}\right]$;

and such that

(c) $F(P)$ is the set of all $J \in \mathcal{J}_{\mathcal{A}}$ such that there is no $J^{\prime} \in \mathcal{J}_{\mathcal{A}}$ with $J^{\prime} \succ_{P} J$. 
Then F satisfies monotonicity.

Proof. Assume $F$ satisfies the conditions of the lemma. Let $P \in \mathcal{J}_{\mathcal{A}}, P^{\prime}$ a $\varphi$ improvement of $P$, and assume that (d) for all $J \in F(P)$ we have $\varphi \in J$.

Let $J^{\prime} \notin F(P)$. From a repeated application of (c), we obtain that there is a $J \in F(P)$ such that $J \succ_{P} J^{\prime}$. From (d), we have $\varphi \in J$. If $\varphi \in J^{\prime}$ then from (a) and (c), we get $J \succ_{P^{\prime}} J^{\prime}$; if $\varphi \notin J^{\prime}$ then from (b) and (c), we get $J \succ_{P^{\prime}} J^{\prime}$; therefore, in all cases, $J^{\prime} \notin F\left(P^{\prime}\right)$. We have shown that $F\left(P^{\prime}\right) \subseteq F(P)$, which together with (d) implies that for all $J \in F\left(P^{\prime}\right)$ we have $\varphi \in J$, from which we conclude that $F$ satisfies monotonicity.

Proposition 7 MC, MCC, MED, RA and LEXIMAX satisfy monotonicity.

Proof. In all cases, the proof comes from an application of Lemma 3, with a suitable family of orders in each case.

- for MC, $\succ_{P}$ is defined by $J \succ_{P} J^{\prime}$ if and only if $J \cap m(P) \supset J^{\prime} \cap m(P)$.

- for MCC, $\succ_{P}$ is defined by $J \succ_{P} J^{\prime}$ if and only if $|J \cap m(P)|>\left|J^{\prime} \cap m(P)\right|$.

- for MED, $\succ_{P}$ is defined by $J \succ_{P} J^{\prime}$ if $\sum_{\psi \in J} N(P, \psi)>\sum_{\psi \in J^{\prime}} N(P, \psi)$.

- for RA, $\succ_{P}$ is $>_{P}^{\mathrm{RA}}$ as defined in Section 3

- for Leximax, $\succ_{P}$ is $>_{P}^{\text {leximax }}$ as defined in Section 3 .

We give the proof that the conditions of the lemma are satisfied for MC (the other cases are similar).

It comes directly from the definition of the rule that (c) holds. Let $P^{\prime}$ be a $\varphi$-improvement of $P$. Then one of the three conditions of Lemma 1 holds.

1. If $m\left(P^{\prime}\right)=m(P)$, then (a) and (b) obviously hold.

2. Assume $\neg \varphi \in m(P)$ and $m\left(P^{\prime}\right)=m(P) \backslash\{\neg \varphi\}$. Let $J, J^{\prime}$ such that $J \succ_{P} J^{\prime}$, that is,

$$
J \cap m(P) \supset J^{\prime} \cap m(P) .
$$

(i) if $\varphi$ belongs to both $J$ and $J^{\prime}$, then $\neg \varphi$ belongs to neither. Then $J \cap m\left(P^{\prime}\right)=J \cap m(P), J^{\prime} \cap m\left(P^{\prime}\right)=J^{\prime} \cap m(P)$, and (9) implies $J \succ_{P^{\prime}} J^{\prime}$.

(ii) if $\neg \varphi$ belongs to both $J$ and $J^{\prime}$, then $J \cap m\left(P^{\prime}\right)=(J \cap m(P)) \backslash\{\neg \varphi\}$ and $J^{\prime} \cap m\left(P^{\prime}\right)=\left(J^{\prime} \cap m(P)\right) \backslash\{\neg \varphi\}$, and then (9) implies $J \cap m\left(P^{\prime}\right) \supset$ $J^{\prime} \cap m\left(P^{\prime}\right)$, that is, $J \succ_{P^{\prime}} J^{\prime}$.

(iii) assume $\varphi$ belongs to $J$ but not to $J^{\prime}$; then $\neg \varphi \notin J$, which together with $\neg \varphi \in m(P)$ and (9) implies $\neg \varphi \notin J^{\prime}$, therefore $\varphi \in J^{\prime}$, contradiction.

3. Assume $\varphi \notin m(P)$ and $m\left(P^{\prime}\right)=(m(P) \backslash\{\neg \varphi\}) \cup\{\varphi\}$. 
(iv) if $\varphi$ belongs to both $J$ and $J^{\prime}$, then $J \cap m\left(P^{\prime}\right)=(J \cap m(P)) \cup\{\varphi\}$ and $J^{\prime} \cap m\left(P^{\prime}\right)=\left(J^{\prime} \cap m(P)\right) \cup\{\varphi\}$. From (9), $\varphi \notin J \cap m(P)$ and $\varphi \notin J^{\prime} \cap m(P)$ we obtain $J \cap m\left(P^{\prime}\right) \supset J^{\prime} \cap m\left(P^{\prime}\right)$, that is, $J \succ_{P^{\prime}} J^{\prime}$.

(v) if $\varphi$ belongs to neither $J$ and $J^{\prime}$, then $J \cap m\left(P^{\prime}\right)=J \cap m(P)$ and $J^{\prime} \cap m\left(P^{\prime}\right) \subseteq J^{\prime} \cap m(P)$, therefore $J \cap m\left(P^{\prime}\right)=J \cap m(P) \subset J^{\prime} \cap$ $m(P) \subseteq J^{\prime} \cap m\left(P^{\prime}\right)$, therefore, $J \succ_{P^{\prime}} J^{\prime}$.

(vi) if $\varphi$ belongs to $J$ but not to $J^{\prime}$, then $J \cap m\left(P^{\prime}\right)=(J \cap m(P)) \cup\{\varphi\}$ and $J^{\prime} \cap m\left(P^{\prime}\right) \subseteq J^{\prime} \cap m(P)$, therefore, $J \cap m\left(P^{\prime}\right)=(J \cap m(P)) \cup\{\varphi\} \supset$ $\left(J^{\prime} \cap m(P)\right) \cup\{\varphi\} \supseteq J^{\prime} \cap m(P)=J^{\prime} \cap m\left(P^{\prime}\right)$, that is, $J \succ_{P^{\prime}} J^{\prime}$.

(i), (ii), (v) and (vi) show that (a) holds in all cases, while (iii) and (vi) show that (b) holds in all cases.

Proposition $8 F^{d_{H}, \operatorname{MAX}}$ and $F^{d_{G}, \Sigma}$ satisfy monotonicity.

Proof. We say that a distance $d$ satisfies agreement monotonicity [32] when for all judgment sets $J, J^{\prime}, J^{\prime \prime} \in \mathcal{J}_{\mathcal{A}},\left(J^{\prime \prime} \backslash J^{\prime}\right) \subset\left(J^{\prime \prime} \backslash J\right)$ implies $d\left(J^{\prime}, J^{\prime \prime}\right) \leq d\left(J, J^{\prime \prime}\right)$. Clearly, $d_{H}$ and $d_{G}$ are agreement monotonic 32]. Let $\star \in\left\{\sum, \max \right\}$. For profile $P=\left(J_{i}, i \in N\right)$ and judgment set $J$, define $d^{\star}(J, P)=\star\left(d\left(J, J_{i}\right) \mid i \in N\right)$, and let $F_{d}^{\star}$ the rule defined by $F_{d}^{\star}(P)=\operatorname{argmin}_{J \in \mathcal{J}_{\mathcal{A}}} d^{\star}(J, P)$.

Let $d \in\left\{d_{H}, d_{G}\right\}$. Let $P=\left(J_{i}, P_{-i}\right)$ be a profile such that $\varphi \in J$ for all $J \in F_{d}^{\star}(P)$, and $P^{\prime}=\left(J_{i}^{\prime}, P_{-i}\right)$ a $\varphi$-improvement of $P$. Let $J \in F_{d}^{\star}(P)$ and $J^{\prime}$ such that $\neg \varphi \in J^{\prime}$. Because $J^{\prime} \notin F_{d}^{\star}(P)$, we have $d^{\star}\left(J^{\prime}, P\right)>d^{\star}(J, P)$. Since $\neg \varphi \in J_{i}, \varphi \in J_{i}^{\prime}$, and $\varphi \in J$, by agreement monotonicity of $d$ we have $d\left(J, J_{i}^{\prime}\right) \leq d\left(J, J_{i}\right)$ and $d\left(J^{\prime}, J_{i}^{\prime}\right) \geq d\left(J^{\prime}, J_{i}\right)$. Therefore,

$$
d^{\star}\left(J^{\prime}, P^{\prime}\right) \geq d^{\star}\left(J^{\prime}, P\right)>d^{\star}(J, P) \geq d^{\star}\left(J, P^{\prime}\right),
$$

which shows that $J^{\prime} \notin F_{d}^{\star}\left(P^{\prime}\right)$. Therefore, $F_{d}^{\star}\left(P^{\prime}\right)$ satisfies monotonicity, and as particular cases, $F^{d_{H}, \text { MAX }}$, MED and $F^{d_{G}, \Sigma}$ satisfy monotonicity.

\section{Proposition 9 y satisfies monotonicity.}

Proof.

Let $P=\left(J_{i}, P_{-i}\right)$, and $P^{\prime}=\left(J_{i}^{\prime}, P_{-i}\right)$ a $\varphi$-improvement of $P$, that is, (1) $P^{\prime}=\left(J_{i}^{\prime}, P_{-i}\right)$, with $J_{i}^{\prime}=\left(J_{i} \backslash\{\neg \varphi\}\right) \cup\{\varphi\}$ (and $J_{i}^{\prime}$ consistent). Assume that (2) every judgment set in $\mathrm{Y}(P)$ contains $\varphi$. Assume as well that (3) some judgment set in $\mathrm{Y}\left(P^{\prime}\right)$ contains $\neg \varphi$, which means that (4) there is a maximum cardinality majority-consistent subprofile $Q^{\prime}$ of $P^{\prime}$ such that $\neg \varphi \in J^{\prime}$ for some $J^{\prime} \in \operatorname{ext}\left(m\left(Q^{\prime}\right)\right)$. We distinguish two cases:

Case 1: $J_{i}^{\prime} \notin Q^{\prime}$. Then $Q^{\prime}$ is also a majority-consistent subprofile of $P$. Because $Q^{\prime}$ can be extended into a judgment set containing $\neg \varphi, Q^{\prime}$ cannot be a maxcard majority-consistent subprofile of $P$, thus (5) there exists a maxcard majorityconsistent subset $U$ of $P$ with $|U|>\left|Q^{\prime}\right|$. 
Case 1.1: $J_{i} \notin U$. Then $U$ is also a majority-consistent subset of $P^{\prime}$ with $|U|>\left|Q^{\prime}\right|$, which contradicts (4).

Case 1.2: $J_{i} \in U$. Let $U^{\prime}=\left(U \backslash\left\{J_{i}\right\}\right) \cup\left\{J_{i}^{\prime}\right\}$. Note that $U^{\prime}$ is a $\varphi$-improvement of $U$. Because of (2) and (5), we have (6) every rational extension of $m(U)$ contains $\varphi$. By point (a) of Lemma 2 applied to $U, \neg \varphi \notin m(U)$. Now, we apply Lemma 1 to $U$. Condition (2) is impossible because $\neg \varphi \notin m(U)$, and condition (3) simplifies to $m\left(U^{\prime}\right)=m(U) \cup\{\varphi\}$; therefore, either $m\left(U^{\prime}\right)=m(U)$ or $m\left(U^{\prime}\right)=m(U) \cup\{\varphi\}$. If $m\left(U^{\prime}\right)=m(U)$ then trivially, $U^{\prime}$ is majority-consistent. If $m\left(U^{\prime}\right)=m(U) \cup\{\varphi\}$ then applying point (b) of Lemma 2 to $J=m(U)$, we obtain that $m(U)$ and $m\left(U^{\prime}\right)$ have the same rational extensions, which in turn implies that $U^{\prime}$ is majority-consistent. Thus, $U^{\prime}$ is a majority-consistent subset of $P^{\prime}$ with $\left|U^{\prime}\right|=|U|>\left|Q^{\prime}\right|$, which contradicts (4).

Case 2: $J_{i}^{\prime} \in Q^{\prime}$. Let $Q=\left(Q^{\prime} \backslash\left\{J_{i}^{\prime}\right\}\right) \cup\left\{J_{i}\right\}$. Because of $(4), m\left(Q^{\prime}\right)$ does not contain $\varphi$, and because $Q^{\prime}$ is a $\varphi$-improvement of $Q, m(Q)$ does not contain $\varphi$ either, and moreover $m(Q)$ and $m\left(Q^{\prime}\right)$ coincide on all issues other than $\varphi, \neg \varphi$. This implies that a rational extension $J^{\prime}$ of $m\left(Q^{\prime}\right)$ containing $\varphi$ is also a rational extension of $m(Q)$, therefore, $m(Q)$ is consistent and has some rational extension containing $\neg \varphi$. This, together with (2), implies that $Q$ cannot be a maxcard majority-consistent subprofile of $P$, that is, there is a maxcard majority-consistent subprofile $T$ of $P$ such that $|T|>|Q|$.

Case 2.1: $J_{i} \notin T$. Then $T$ is also a majority-consistent subprofile of $P^{\prime}$, and $|T|>|Q|=\left|Q^{\prime}\right|$, which contradicts (4).

Case 2.2: $J_{i} \in T$. Let $T^{\prime}=\left(T \backslash\left\{J_{i}\right\}\right) \cup\left\{J_{i}^{\prime}\right\}$. Similarly as in case 1.2, $m(T)$ and $m\left(T^{\prime}\right)$ have the same rational extensions, and $T^{\prime}$ is a majority-consistent subprofile of $P^{\prime}$ such that $\left|P^{\prime}\right|>\left|Q^{\prime}\right|$, which contradicts (4).

Here come now two rather surprising results.

Proposition 10 Frev does not satisfy monotonicity.

Proof. Consider an agenda $\mathcal{A}=\left\{p_{1}, \neg p_{1}, \ldots, p_{14}, \neg p_{14}\right\}$ and constraint $\gamma$, such that $\mathcal{J}_{\mathcal{A}}$ contains judgment sets $J^{1}$ to $J^{29}$ as given in Table 11]

Consider the profile of two agents $P=\left\langle J_{1}, J_{2}\right\rangle$ where $J_{1}=J^{1}$ and $J_{2}=$ $J^{14}$. We obtain that $F_{\operatorname{rev}}(P)=\left\{J^{8}, J^{9}, J^{10}, J^{11}\right\}$. Observe that for every $J \in F_{\text {rev }}(P), \neg p_{1} \in J$. Consider the $\neg p_{1}$-improvement of $P$, the profile $P^{\prime}=\left\langle J_{1}^{\prime}, J_{2}\right\rangle$, where $J_{1}^{\prime}=J^{2}$. We now have that $F_{\mathrm{rev}}\left(P^{\prime}\right)=\left\{J^{29}\right\}$ and $\neg p_{1} \notin J^{29}$.

Thus, $F_{\text {rev }}$ fails to satisfy monotonicity although, of course, the Borda rule does satisfy it 8

\footnotetext{
${ }^{8}$ This surprising result triggers further questions: are there interesting agendas, other than the preference agenda, for which $F_{\text {rev }}$ remains monotonic? Can we find a natural monotonic extension of the Borda rule? Such intriguing questions are left for further study.
} 


\begin{tabular}{|c|c|c|c|c|c|c|c|c|c|c|c|c|c|c|}
\hline Sets & $\left\{p_{1}\right.$ & $p_{2}$, & $p_{3}$, & $p_{4}$, & $p_{5}$, & $p_{6}$, & $p_{7}$, & $p_{8}$, & $p_{9}$, & $p_{10}$, & $p_{11}$, & $p_{12}$, & $p_{13}$, & $\left.p_{14}\right\}$ \\
\hline$J^{1}$ & + & + & + & + & + & + & + & + & + & + & + & + & + & + \\
\hline$J^{2}$ & - & + & + & + & + & + & + & + & + & + & + & + & + & + \\
\hline$J^{3}$ & - & - & + & + & + & + & + & + & + & + & + & + & + & + \\
\hline$J^{4}$ & - & + & - & + & + & + & + & + & + & + & + & + & + & + \\
\hline$J^{5}$ & - & + & + & - & + & + & + & + & + & + & + & + & + & + \\
\hline$J^{6}$ & - & + & + & + & - & + & + & + & + & + & + & + & + & + \\
\hline$J^{7}$ & - & + & + & + & + & - & + & + & + & + & + & + & + & + \\
\hline$J^{8}$ & - & + & + & + & + & + & - & + & + & + & + & + & + & + \\
\hline$J^{9}$ & - & + & + & + & + & + & + & - & + & + & + & + & + & + \\
\hline$J^{10}$ & - & + & + & + & + & + & + & + & - & + & + & + & + & + \\
\hline$J^{11}$ & - & + & + & + & + & + & + & + & + & - & + & + & + & + \\
\hline$J^{12}$ & + & + & + & + & + & + & + & + & + & + & - & - & + & + \\
\hline$J^{13}$ & + & + & + & + & + & + & + & + & + & + & + & + & - & - \\
\hline$J^{14}$ & - & - & - & - & - & - & - & - & - & - & - & - & - & - \\
\hline$J^{15}$ & + & + & - & - & - & - & - & - & - & - & - & - & - & - \\
\hline$J^{16}$ & - & + & - & - & - & - & - & - & - & - & - & - & - & - \\
\hline$J^{17}$ & - & - & + & - & - & - & - & - & - & - & - & - & - & - \\
\hline$J^{18}$ & - & - & - & + & - & - & - & - & - & - & - & - & - & - \\
\hline$J^{19}$ & - & - & - & - & + & - & - & - & - & - & - & - & - & - \\
\hline$J^{20}$ & - & - & - & - & - & + & - & - & - & - & - & - & - & - \\
\hline$J^{21}$ & - & - & - & - & - & - & + & - & - & - & - & - & + & + \\
\hline$J^{22}$ & - & - & - & - & - & - & - & + & - & - & - & - & + & + \\
\hline$J^{23}$ & - & - & - & - & - & - & - & - & + & - & - & - & + & + \\
\hline$J^{24}$ & - & - & - & - & - & - & - & - & - & + & - & - & + & + \\
\hline$J^{25}$ & - & - & - & - & - & - & - & - & - & - & + & - & - & - \\
\hline$J^{26}$ & - & - & - & - & - & - & - & - & - & - & - & + & - & - \\
\hline$J^{27}$ & - & - & - & - & - & - & - & - & - & - & - & - & + & - \\
\hline$J^{28}$ & - & - & - & - & - & - & - & - & - & - & - & - & - & + \\
\hline$J^{29}$ & + & - & - & - & - & - & - & - & - & - & - & + & + & + \\
\hline
\end{tabular}

Table 11: Counterexample showing that $F_{\text {rev }}$ does not satisfying monotonicity.

Proposition 11 MPC does not satisfy monotonicity.

Proof. Consider an agenda $\mathcal{A}=\left\{p_{1}, \neg p_{1}, \ldots, p_{16}, \neg p_{16}\right\}$ and constraint $\gamma$, such that $\mathcal{J}_{\mathcal{A}}=\left\{J^{1}, \ldots, J^{9}\right\}$ as given in Table 12,

Consider profile $P=\left\langle J^{1}, J^{2}, J^{3}\right\rangle$. Let $Q=\left\langle J^{1}, J^{2}, J^{8}\right\rangle$. We observe that $d(P, Q)=d\left(J^{3}, J^{8}\right)=5$, and that $m(Q)=\left\{J_{4}\right\}$, therefore $Q$ is majorityconsistent. We claim that there is no other majority-consistent profile $Q^{\prime}$ with $d\left(P, Q^{\prime}\right) \leq 5$. To check this, we first give the distances between $J^{1}, J^{2}, J^{3}$ and other judgement sets; they are represented in Table 13.

Now, we list all profiles such that $d\left(P, Q^{\prime}\right) \leq 5$. There are 10; they are shown in Table 14, together with their distance to $P$ and their majoritarian aggregation. All of them except $Q$ are majority-inconsistent. Therefore, $\operatorname{MPC}(P)=m(Q)=\left\{J^{4}\right\}$. Also, observe that $p_{1} \in J^{4}$.

Consider now $P^{\prime}=\left\langle J^{1}, J^{2}, J^{9}\right\rangle$. Since $J^{3}$ and $J^{9}$ differ only on $p_{1}, P^{\prime}$ is a $p_{1}$-improvement of $P$. Now, we claim that $\operatorname{MPC}\left(P^{\prime}\right)=\left\{J^{4}, J^{5}\right\}$. First, 


\begin{tabular}{c|c|ccc|ccc|ccc|ccc|ccc} 
& $p_{1}$ & $p_{2}$ & $p_{3}$ & $p_{4}$ & $p_{5}$ & $p_{6}$ & $p_{7}$ & $p_{8}$ & $p_{9}$ & $p_{10}$ & $p_{11}$ & $p_{12}$ & $p_{13}$ & $p_{14}$ & $p_{15}$ & $p_{16}$ \\
\hline$J^{1}$ & + & + & + & + & + & + & + & + & + & + & + & + & + & + & + & + \\
$J^{2}$ & + & + & - & - & + & - & - & + & - & - & + & - & - & + & - & - \\
$J^{3}$ & - & - & + & - & - & + & - & - & + & - & - & + & - & - & + & - \\
\hline$J^{4}$ & + & + & + & - & + & - & + & + & - & - & + & + & - & + & + & - \\
$J^{5}$ & - & + & + & - & + & + & - & + & + & - & + & + & - & + & + & - \\
$J^{6}$ & - & + & - & - & + & + & + & + & + & + & + & + & + & + & + & + \\
$J^{7}$ & - & + & + & + & + & - & - & + & - & - & + & - & - & + & - & - \\
$J^{8}$ & - & - & + & - & + & - & + & + & - & - & - & + & - & - & + & - \\
\hline$J^{9}$ & + & - & + & - & - & + & - & - & + & - & - & + & - & - & + & -
\end{tabular}

Table 12: Counter-example showing that MPC does not satisfy monotonicity.

\begin{tabular}{c|c|c|c|c|c|c|c|c|c} 
& $J^{1}$ & $J^{2}$ & $J^{3}$ & $J^{4}$ & $J^{5}$ & $J^{6}$ & $J^{7}$ & $J^{8}$ & $J^{9}$ \\
\hline$J^{1}$ & 0 & 10 & 11 & 6 & 6 & 3 & 9 & 10 & 10 \\
$J^{2}$ & 10 & 0 & 11 & 4 & 6 & 9 & 3 & 8 & 10 \\
$J^{3}$ & 11 & 11 & 0 & 9 & 5 & 10 & 10 & 5 & 1
\end{tabular}

Table 13: Distances between $J^{1}, J^{2}, J^{3}$ and other judgement sets.

we observe that $d\left(P^{\prime}, Q\right)=d\left(J^{9}, J^{8}\right)=6$. We show now that there are no majority-consistent profile $Q^{\prime}$ with $d\left(P^{\prime}, Q^{\prime}\right)<6$, and that there is another one with $d\left(P^{\prime}, Q^{\prime}\right)=6$. To check this, we first give the distances between $J_{9}$ and each of the 9 consistent judgment sets, which are shown in Table 15.

Let us now list all profiles such that $d\left(P^{\prime}, Q^{\prime}\right) \leq 6$. There are 14 of them: all those that satisfied $d\left(P, Q^{\prime}\right) \leq 5$, and four more that are shown in Table 16 .

We conclude that there are exactly two consistent profiles at distance 6 from $P^{\prime}: Q$ and $\left\langle J^{6}, J^{7}, J^{9}\right\rangle$. Therefore, $\operatorname{mPC}\left(P^{\prime}\right)=\left\{J^{4}, J^{5}\right\}$. Now, $\neg p_{1} \in J^{5}$, which shows that MPC does not satisfy monotonicity.

\begin{tabular}{|c|c|c|c|c|c|c|}
\hline$Q^{\prime}$ & $d\left(P, Q^{\prime}\right)$ & \multicolumn{5}{|c|}{$\operatorname{maj}\left(Q^{\prime}\right)$} \\
\hline$P$ & 0 & +++- & ++- & ++- & ++- & ++- \\
\hline$Q$ & 5 & & & $J_{4}$ & & \\
\hline$\left\langle J^{1}, J^{2}, J^{5}\right\rangle$ & 5 & +++- & ++- & ++- & ++- & ++- \\
\hline$\left\langle J^{1}, J^{2}, J^{9}\right\rangle$ & 1 & +++- & ++- & ++- & ++- & ++- \\
\hline$\left\langle J^{1}, J^{7}, J^{3}\right\rangle$ & 3 & -+++ & ++- & ++- & ++- & ++- \\
\hline & 4 & ++++ & ++- & ++- & ++- & ++- \\
\hline$\left\langle J^{1}, J^{4}, J^{3}\right\rangle$ & 4 & +++- & +++ & ++- & ++- & ++- \\
\hline$\left\langle J^{1}, J^{4}, J^{9}\right\rangle$ & 5 & +++- & +++ & ++- & ++- & ++- \\
\hline$\left\langle J^{6}, J^{2}, J^{3}\right\rangle$ & 3 & -+-- & ++- & ++- & ++- & ++- \\
\hline$\left\langle J^{6}, J^{2}, J^{9}\right\rangle$ & 4 & ++-- & ++- & ++- & ++- & ++- \\
\hline
\end{tabular}

Table 14: All the profiles at distances 5 or less from $P$. 


\begin{tabular}{c|c|c|c|c|c|c|c|c|c} 
& $J^{1}$ & $J^{2}$ & $J^{3}$ & $J^{4}$ & $J^{5}$ & $J^{6}$ & $J^{7}$ & $J^{8}$ & $J^{9}$ \\
\hline$J^{9}$ & 10 & 10 & 1 & 8 & 6 & 11 & 11 & 6 & 0
\end{tabular}

Table 15: Distances between $J_{9}$ and each of the 9 consistent judgment sets.

\begin{tabular}{|c|c|c|}
\hline$Q^{\prime}$ & $d\left(P^{\prime}, Q^{\prime}\right)$ & $\operatorname{maj}\left(Q^{\prime}\right)$ \\
\hline$\left\langle J^{6}, J^{7}, J^{9}\right\rangle$ & 6 & $J^{5}$ \\
\hline$\left\langle J^{4}, J^{2}, J^{9}\right\rangle$ & 6 & +++-+--+--++-++- \\
\hline$\left\langle J^{1}, J^{5}, J^{9}\right\rangle$ & 6 & +++-++-++-++-++- \\
\hline$\left\langle J^{5}, J^{2}, J^{9}\right\rangle$ & 6 & +++-++-++-++-++- \\
\hline
\end{tabular}

Table 16: There are 14 profiles at distance 6 or less from $P^{\prime}$ : four profiles from this table and all those from Table 14.

\subsection{Reinforcement}

A social preference function $F$ satisfies reinforcement if whenever two profiles over disjoint electorates have some output rankings in common, then the profiles obtained by merging the two electorates leads to elect those rankings that are obtained for both profiles. This easily generalizes to judgment aggregation rules as follows.

Definition 12 For every two profiles $P=\left\langle J_{1}, \ldots, J_{n}\right\rangle$ and $Q=\left\langle J_{n+1}, \ldots, J_{q}\right\rangle$, we denote $P+Q=\left\langle J_{1}, \ldots, J_{q}\right\rangle$. We say that a judgment aggregation rule $F$ satisfies reinforcement when for every agenda $\mathcal{A}$, and every two profiles $P$ and $Q$ over disjoint electorates, if $F(P) \cap F(Q) \neq \emptyset$ then $F(P+Q)=F(P) \cap F(Q)$.

Young and Levenglick's theorem [35] tells us that among social preference functions Kemeny's rule is the unique Condorcet extension satisfying neutrality and reinforcement. As a consequence, if a judgment aggregation rule is majoritypreserving and if its application to a preference agenda defines a neutral social preference function, then this SPF has to be Kemeny's rule.

Corollary 2 MC, MCC, RA, LEXIMAX, MPC and Y do not satisfy reinforcement.

The following result does not come as a surprise, as reinforcement is the key property of scoring voting rules:

Proposition 12 All scoring rules satisfy reinforcement.

Proof. Let $F_{s}$ be the scoring rule based on some scoring function $s$. Let $P$ and $Q$ be two profiles over disjoint electorates and assume that $F_{s}(P) \cap F_{s}(Q) \neq \emptyset$. Let $J \in F_{s}(P) \cap F_{s}(Q)$. Then $s(J, P) \geq s\left(J^{\prime}, P\right)$ and $s(J, Q) \geq s\left(J^{\prime}, Q\right)$ for all $J^{\prime}$, therefore, $s(J, P+Q)=s(J, P)+s(J, Q) \geq s\left(J^{\prime}, P\right)+s\left(J^{\prime}, Q\right)=s\left(J^{\prime}, P+Q\right)$, 
which shows that $J \in F_{s}(P+Q)$. Conversely, let $J^{\prime} \notin F_{s}(P) \cap F_{s}(Q)$. Without loss of generality, assume $J^{\prime} \notin F_{s}(P)$. Then, for every $J \in F_{s}(P) \cap F_{s}(Q)$ we have $s(J, P)>s\left(J^{\prime}, P\right)$, therefore $s\left(J^{\prime}, P+Q\right)=s\left(J^{\prime}, P\right)+s\left(J^{\prime}, Q\right)>$ $s(J, P)+s(J, Q)=s(J, P+Q)$, which shows that $J^{\prime} \notin F_{s}(P+Q)$.

Corollary 3 MED and Frev satisfy reinforcement.

Proposition $13 F^{d_{G}, \Sigma}$ satisfies reinforcement.

The proof is similar to the proof above for scoring rules, replacing scores by distances and maximization by minimization. It would work more generally for every rule minimising the sum of distances to judgment sets.

Proposition $14 F^{d_{H}, \mathrm{MAX}}$ does not satisfy reinforcement.

Proof. Let $[\mathcal{A}]=\{p, q, r\}, \gamma=\top, P=\langle\{p, q, r\},\{\neg p, \neg q, \neg r\}\rangle$ and $Q=$ $\langle\{\neg p, q, r\}\rangle . \quad F^{d_{H}, \operatorname{MAX}}(P)=\{\{\neg p, q, r\},\{p, \neg q, r\},\{p, q, \neg r\},\{\neg p, \neg q, r\}$, $\{\neg p, q, \neg r\},\{\neg p, \neg q, r\}\}$ and $F^{d_{H}, \operatorname{MAx}}(Q)=\{\{\neg p, q, r\}\}$, therefore $F^{d_{H}, \operatorname{MAx}}(P) \cap F^{d_{H}, \operatorname{MAx}}(Q)=F^{d_{H}, \operatorname{MAx}}(Q) \neq \emptyset$. However, $F^{d_{H}, \operatorname{MAx}}(P+Q)=$ $F^{d_{H}, \operatorname{MAX}}(P)$.

A similar negative result holds more generally for every rule minimising the maximum of distances to judgment sets. However, such rules, including $F^{d_{H}, \text { MAx }}$, satisfy this weak version of reinforcement: if $F^{d_{H}, \operatorname{MAx}}(P) \cap F^{d_{H}, \operatorname{MAx}}(Q) \neq \emptyset$, then $F^{d_{H}, \operatorname{MAx}}(P+Q) \cap\left(F^{d_{H}, \operatorname{MAx}}(P) \cap F^{d_{H}, \operatorname{MAx}}(Q)\right) \neq \emptyset$.

\subsection{Homogeneity}

Let us write $k P$ for $\underbrace{P+\cdots+P}_{k \text { times }}$, where + has been defined in Subsection 6.3 ,

Definition 13 A judgment aggregation rule $F$ satisfies homogeneity when for every $\mathcal{J}_{\mathcal{A}}$-profile $P$ and positive integer $k$, it holds that $F(k P)=F(P)$.

Homogeneity being weaker than reinforcement, we already know that it is satisfied by all scoring functions (including MED and $F_{\text {rev }}$ ) and by $F^{d_{G}, \Sigma}$.

Proposition 15 Every judgment aggregation rule based on the majority set satisfies homogeneity.

Proof. Let $F$ be a judgment aggregation rule based on majority set. Since for every profile $P$ and every $k \in\{1,2,3, \ldots\}$ we have that $m(P)=m(k P)$ then $F(P)=F(k P)$.

Corollary 4 MC and MCC satisfy homogeneity. 
Proposition 16 RA and LEXIMAX satisfy homogeneity.

Proof. $\operatorname{RA}(P)$ is fully determined by the weak order on $\mathcal{A}$ induced by the values of $N(P,$.$) . Since for every k \in\{1,2, \ldots\}$ and every $\varphi, \psi \in \mathcal{A}, N(k P, \varphi) \geq N(k P, \psi)$ if and only if $N(P, \varphi) \geq N(P, \psi)$, we have $\operatorname{RA}(P)=\operatorname{RA}(k P)$. The same proof works also for LEXIMAX.

Proposition 17 Y does not satisfy homogeneity.

Proof. This is a consequence of the fact that the Young voting rule does not satisfy homogeneity (see Example 2 in [34]).

Proposition 18 MPC does not satisfy homogeneity.

Proof. Consider the profile $P$ from Table 17. We have $\operatorname{MPc}(P)=\{\{\neg(p \wedge$

\begin{tabular}{r|cccc} 
Voters & $p \wedge r$ & $p \wedge s$ & $q$ & $p \wedge q$ \\
\hline$J_{1}, J_{2}, J_{3}$ & + & + & + & + \\
$J_{4}, J_{5}, J_{6}$ & + & + & - & - \\
$J_{7}-J_{10}$ & - & - & + & - \\
$J_{11}$ & - & - & - & - \\
\hline$m(P)$ & + & + & + & -
\end{tabular}

Table 17: Profile showing that MPC does not satisfy homogeneity.

$r), \neg(p \wedge s), q, \neg(p \wedge q)\},\{(p \wedge r),(p \wedge s), \neg q, \neg(p \wedge q)\}\}$. Consider now the profile $P^{\prime}=2 P=P+P$. We have $\operatorname{MPC}\left(P^{\prime}\right)=\{\{\neg(p \wedge r), \neg(p \wedge s), q, \neg(p \wedge q)\}\}$.

Proposition 19 For every distance d, $F^{d, \max }$ satisfies homogeneity.

Proof. For every profile $P$, judgment set $J$, and positive integer $k$, we have $\max _{J_{i} \in k P} d\left(J, J_{i}\right)=\max _{J_{i} \in P} d\left(J, J_{i}\right)$. The result follows.

As a consequence, $F^{d_{H}, \max }$ satisfies homogeneity.

\section{$7 \quad$ Summary}

We have listed a number of existing judgment aggregation rules, and for a number of important properties we have identified those rules that satisfy it. These properties come in four groups: (1) majority-preservation; (2) weak and strong unanimity; (3) monotonicity, and (4) reinforcement and homogeneity (weaker than reinforcement). Our results are summarized in Table 18 , 


\begin{tabular}{|l|c|c|c|c|c|c|c|c|c|c|}
\hline Property & MC & MCC & RA & LEXIMAX & MED & MPC & Y & $F_{G}^{d_{G}, \Sigma}$ & $F^{d_{H}, \text { MAX }}$ & $F_{\text {rev }}$ \\
\hline Majority Preservation & yes & yes & yes & yes & yes & yes & yes & no & no & no \\
\hline Weak Unanimity & yes & no & yes & yes & no & no & yes & no & no & no \\
\hline Strong Unanimity & no & no & yes & yes & no & no & yes & no & no & no \\
\hline Monotonicity & yes & yes & yes & yes & yes & no & yes & yes & yes & no \\
\hline Homogeneity & yes & yes & yes & yes & yes & no & no & yes & yes & yes \\
\hline Reinforcement & no & no & no & no & yes & no & no & yes & no* & yes \\
\hline
\end{tabular}

Table 18: Summary of rules and properties they (do not) satisfy.

We may use this table to derive a tentative dominance relation between rules. Let $\mathcal{P}$ be the set of properties considered here, that is, $\mathcal{P}=$ \{majority preservation, weak unanimity, strong unanimity, monotonicity, reinforcement, homogeneity $\}$. Say that a rule $F \mathcal{P}$-dominates a rule $F^{\prime}$ when the set of properties in $\mathcal{P}$ satisfied by $F$ strictly contains the set of properties in $\mathcal{P}$ satisfied by $F^{\prime}$. (Of course, this is somewhat arbitrary because many other properties could have been considered; but still, these are among the most important properties.) Then:

- RA and LEXIMAX $\mathcal{P}$-dominate MC, MCC, MPC and Y;

- MED $\mathcal{P}$-dominates MCC, $F_{\text {rev }}, \operatorname{MPC}, F^{d_{G}, \Sigma}$ and $F^{d_{H}, \mathrm{MAx}}$.

This leaves us with three $\mathcal{P}$-undominated rules, coming in two groups: MED, and the very closely related rules RA and LEximax. Given the importance of the median rule, which generalizes the Kemeny rule, it should not come as a surprise that MED performs well. The presence of RA and LEXIMAX on this podium is somewhat more surprising.

Some rules have been left out of this study. Importantly, we did not consider quota-based rules, because the quota has to be chosen depending on the agenda to ensure that the judgment sets are consistent, which prevents the use of a quota-based rule in an agenda-independent way. This is even more patent for premise-based and conclusion-based rules.

Finally, a challenging question is the axiomatization of some judgment aggregation rules, or some families of rules, for which our work can be seen as a very first (and very incomplete) step?

\section{Acknowledgments}

Gabriella Pigozzi and Srdjan Vesic benefited from the support of the project AMANDE ANR-13-BS02-0004 of the French National Research Agency (ANR). Jérôme Lang benefited from the support of the ANR project 14-CE24-0007-01 CoCoRICo-CoDec. The authors would like to thank Denis Bouyssou, as well as anonymous reviewers.

\footnotetext{
${ }^{9}$ There exists a recent axiomatization of the median rule (in the general judgement aggregation framework) 26]; we are not aware of axiomatic characterizations of other rules.
} 


\section{References}

[1] Baumeister, D., Erdélyi, G., Rothe, J.: Judgment aggregation. In: J. Rothe (ed.) Economics and Computation, chap. 6, pp. 361-394. Springer (2016)

[2] Brams, S., Fishburn., P.: Chapter 4: Voting procedures. In: K. Arrow, A. Sen, K. Suzumura (eds.) Handbook of Social Choice and Welfare. Elsevier (2004)

[3] Dietrich, F.: Scoring rules for judgment aggregation. Social Choice and Welfare 42(4), 873-911 (2014)

[4] Dietrich, F., List, C.: Arrow's theorem in judgment aggregation. Social Choice and Welfare 29(1), 19-33 (2007)

[5] Dietrich, F., List, C.: Judgment aggregation by quota rules: Majority voting generalized. Journal of Theoretical Politics 4(19), 391 - 424 (2007)

[6] Dietrich, F., List, C.: Strategy-proof judgment aggregation. Economics and Philosophy 23, 269-300 (2007)

[7] Dietrich, F., List, C.: A liberal paradox for judgment aggregaton. Social Choice and Welfare 31(1), 59-78 (2008)

[8] Dietrich, F., Mongin, P.: The premisse-based approach to judgment aggregation. Journal of Economic Theory 145(2), 562-582 (2010)

[9] Duddy, C., Piggins, A.: A measure of distance between judgment sets. Social Choice and Welfare 39(4), 855-867 (2012)

[10] Duddy, C., Piggins, A., Zwicker, W.: Aggregation of binary evaluations: a Borda-like approach. Social Choice and Welfare (2015)

[11] Elkind, E., Faliszewski, P., Slinko, A.: On distance rationalizability of some voting rules. In: Proceedings of the 12th Conference on Theoretical Aspects of Rationality and Knowledge, pp. 108-117 (2009)

[12] Endriss, U.: Judgment aggregation. In: F. Brandt, V. Conitzer, U. Endriss, J. Lang, A.D. Procaccia (eds.) Handbook of Computational Social Choice, chap. 17. Cambridge University Press (2016)

[13] Endriss, U., Grandi, U., de Haan, R., Lang, J.: Succinctness of languages for judgment aggregation. In: Principles of Knowledge Representation and Reasoning: Proceedings of the Fifteenth International Conference, KR 2016, Cape Town, South Africa, April 25-29, 2016., pp. 176-186 (2016)

[14] Endriss, U., Grandi, U., Porello, D.: Complexity of judgment aggregation. Journal of Artificial Intelligence Research 45, 481-514 (2012)

[15] Everaere, P., Konieczny, S., Marquis, P.: Counting votes for aggregating judgments. In: International conference on Autonomous Agents and MultiAgent Systems, pp. 1177-1184 (2014) 
[16] Everaere, P., Konieczny, S., Marquis, P.: Belief merging versus judgment aggregation. In: Proceedings of the 2015 International Conference on $\mathrm{Au}-$ tonomous Agents and Multiagent Systems, AAMAS 2015, Istanbul, Turkey, May 4-8, 2015, pp. 999-1007 (2015)

[17] Grandi, U., Endriss, U.: Lifting integrity constraints in binary aggregation. Artif. Intell. 199, 45-66 (2013)

[18] Grossi, D., Pigozzi, G.: Judgment Aggregation: A Primer. Synthesis Lectures on Artificial Intelligence and Machine Learning. Morgan \& Claypool Publishers (2014). DOI 10.2200/S00559ED1V01Y201312AIM027. URL http://dx.doi.org/10.2200/S00559ED1V01Y201312AIM027

[19] Konieczny, S., Pino-Pérez, R.: Merging information under constraints: a logical framework. Journal of Logic and Computation 12(5), 773-808 (2002)

[20] Lang, J.: Twenty-five years of preferred subtheories. In: T. Eiter, H. Strass, M. Truszczynski, S. Woltran (eds.) Advances in Knowledge Representation, Logic Programming, and Abstract Argumentation - Essays Dedicated to Gerhard Brewka on the Occasion of His 60th Birthday, Lecture Notes in Computer Science, vol. 9060, pp. 157-172. Springer (2015)

[21] Lang, J., Pigozzi, G., Slavkovik, M., van der Torre, L.: Judgment aggregation rules based on minimization. In: Theoretical Aspects of Rationality and Knowledge, pp. 238-246 (2011)

[22] Lang, J., Slavkovik, M.: Judgment aggregation rules and voting rules. In: Proceedings of the 3rd International Conference on Algorithmic Decision Theory, Lecture Notes in Artificial Intelligence, vol. 8176, pp. 230-244. Springer-Verlag (2013)

[23] List, C., Pettit, C.: Aggregating sets of judgments: Two impossibility results compared. Synthese 104(1-2), 207-235 (2004)

[24] List, C., Puppe, C.: Judgment aggregation: A survey. In: P. Anand, C. Puppe, P. Pattanaik (eds.) Oxford Handbook of Rational and Social Choice. Oxford (2009)

[25] Miller, M., Osherson, D.: Methods for distance-based judgment aggregation. Social Choice and Welfare 32(4), 575-601 (2009)

[26] Nehring, K., Pivato, M.: The median rule in judgment aggregation. Tech. rep.

[27] Nehring, K., Pivato, M.: Majority rule in the absence of a majority. MPRA Paper 46721, University Library of Munich, Germany (2013). URL http://ideas.repec.org/p/pra/mprapa/46721.html 
[28] Nehring, K., Pivato, M., Puppe, C.: The Condorcet set: Majority voting over interconnected decisions. Journal of Economic Theory 151, 268-303 (2014)

[29] Nehring, K., Pivato, M., Puppe, C.: Unanimity overruled: Majority voting and the burden of history. Journal of Theoretical Politics (2015). DOI $10.1177 / 0951629815586884$

[30] Pigozzi, G.: Belief merging and the discursive dilemma: an argument-based account to paradoxes of judgment aggregation. Synthese 152(2), 285-298 (2006)

[31] Pigozzi, G., Slavkovik, M., van der Torre, L.: A complete conclusion-based procedure for judgment aggregation. In: F. Rossi, A. Tsoukiàs (eds.) 1rst International Conference on Algorithmic Decision Theory, Venice, Italy, October 20-23, 2009. Proceedings, Lecture Notes in Computer Science, vol. 5783, pp. 1-13. Springer (2009)

[32] Slavkovik, M., Ågotnes, T.: Measuring dissimilarity between judgment sets. In: Logics in Artificial Intelligence, Lecture Notes in Computer Science, vol. 8761, pp. 609-617. Springer International Publishing (2014)

[33] Tideman, T.N.: Independence of clones as a criterion for voting rules. Social Choice and Welfare 4(3), 185-206 (1987)

[34] Young, H.: Extending Condorcet's rule. Journal of Economic Theory 16(2), 335-353 (1977)

[35] Young, H., Levenglick, A.: A consistent extension of Condorcet's election principle. SIAM Journal on Applied Mathematics 2(35), 285-300 (1978)

[36] Zwicker, W.: Towards a Borda count for judgment aggregation (2011). Working Paper.

[37] Zwicker, W.: Voting: an introduction. In: Handbook of Computational Social Choice. Oxford University Press (2014)

\section{Appendix: Proof of Proposition 3.}

Many of the non-inclusion relationships can be derived from the profile of our running example, introduced in Example 2, and used again in Example 3 for MCC and MC, Example 4 for MED, Example 5 for RA and LEximax, Example [6 for $\mathrm{Y}$, and Example 7 for MPC. This profile already shows that MC $\nsubseteq$ MCC,

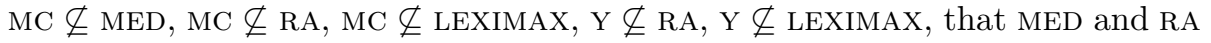
are incomparable, as well as MED and LEXIMAX, that $\mathrm{Y}$ and each of MC, MCC, MED are incomparable, and that MPC is incompatible with each of Y, RA, and LEXIMAX.

The inclusion relationships MCC $\subseteq$ MC, LEXIMAX $\subseteq$ RA are clear from their definitions, and a proof that MED $\subseteq$ MC can be found in 28 . 
We now prove what remains to be proven:

1. RA $\subseteq$ MC: If $J \in \mathrm{RA}(P)$ then, by definition of RA, $J \cap m(P)$ is a maximal consistent subset of $m(P)$, thus $J \in \operatorname{MC}(P)$.

2. Ra $\nsubseteq$ Leximax: Consider the profile $P$ in Table 19 .

\begin{tabular}{r|cccccc} 
Voters & $p \wedge q$ & $p$ & $q$ & $p \wedge r$ & $q \wedge r$ & $s$ \\
\hline$J_{1}-J_{5}$ & - & + & - & + & - & + \\
$J_{6}-J_{10}$ & - & - & + & - & + & - \\
$J_{11}-J_{14}$ & + & + & + & + & + & + \\
$J_{15}$ & + & + & + & - & - & - \\
\hline$m(P)$ & - & + & + & + & + & +
\end{tabular}

Table 19: A profile showing that RA $\not \subset$ LEXIMAX.

$\operatorname{RA}(P)=\{\{p \wedge q, p, q, p \wedge r, q \wedge r, s\},\{\neg(p \wedge q), p, \neg q, p \wedge r, \neg(q \wedge r), s\}\{\neg(p \wedge$ $q), \neg p, q, \neg(p \wedge r), q \wedge r, s\}\}$ and LEXIMAX $(P)=\{\{p \wedge q, p, q, p \wedge r, q \wedge r, s\}\}$.

3. MED $\nsubseteq$ MCC: Consider the example from Table 6. We have $\operatorname{MCC}(P)=$ $\{\neg p, p \rightarrow(q \vee r), \neg p, \neg r, p \rightarrow(s \vee t), \neg s, \neg t, p \rightarrow(u \vee v), \neg u, \neg v\}$ and $\{p, p \rightarrow(q \vee r), \neg q, r, p \rightarrow(s \vee t), \neg s, t, p \rightarrow(u \vee v), \neg u, v\} \in \operatorname{MED}(P)$

4. RA and LEXIMAX are incomparable with MCC.

Consider again the example from Table 6. $\operatorname{MCC}(P)=\{\{\neg p, p \rightarrow(q \vee$ $r), \neg p, \neg r, p \rightarrow(s \vee t), \neg s, \neg t\}, p \rightarrow(u \vee v), \neg u, \neg v\}$ and for every $J \in$ $\operatorname{RA}(P)$, and a fortiori for every $J \in \operatorname{RA}(P), p \in J$. Thus MCC $\nsubseteq$ RA and LEXIMAX $\nsubseteq$ MCC.

5. RA $\nsubseteq \mathrm{Y}$ : Consider the example from Table 20. The minimal number of

\begin{tabular}{|c|c|c|c|c|c|c|c|c|}
\hline Voters & $\{p$ & $q$, & $p \wedge q$ & $r$ & $s$ & $r \wedge$ & $s$ & $t\}$ \\
\hline$J_{1}$ & + & + & + & - & + & - & & + \\
\hline$J_{2}, J_{3}, J_{4}$ & + & + & + & - & + & - & & - \\
\hline$J_{5}-J_{8}$ & + & + & + & + & - & - & & - \\
\hline$J_{9}, J_{10}$ & + & - & - & + & - & - & & - \\
\hline$J_{11}-J_{14}$ & + & - & - & + & + & + & & + \\
\hline$J_{15}-J_{18}$ & - & + & - & + & + & + & & + \\
\hline Rule & $\{p$, & $q$, & $p \wedge q$, & $r$, & $s$, & $r \wedge$ & $s$, & $t\}$ \\
\hline \multirow[t]{2}{*}{$\mathrm{RA}(P)$} & + & + & + & + & + & + & & + \\
\hline & + & + & + & + & + & + & & - \\
\hline $\mathrm{Y}(P)$ & + & + & + & + & + & + & & + \\
\hline
\end{tabular}

Table 20: A profile showing that RA $\nsubseteq$ Y.

voters to remove to make the profile majority-consistent is two. These two voters are the two voters of the fourth row (light gray shaded). We have $\mathrm{Y}(P)=\{\{p, q, p \wedge q, r, s, r \wedge s, t\}\}$ and $\operatorname{RA}(P)=\{\{p, q, p \wedge q, r, s, r \wedge$ $s, t\},\{p, q, p \wedge q, r, s, r \wedge s, \neg t\}\}$. Thus, RA $\nsubseteq \mathrm{Y}$. 
6. MPC is incomparable with MC: Consider the pre-agenda $[\mathcal{A}]=\{p, q, p \wedge$ $\left.q, p \wedge \neg q \wedge r, \alpha_{1}, \alpha_{2}, \neg p \wedge q \wedge s, \alpha_{3}, \alpha_{4}, \alpha_{5}, \alpha_{6}, \alpha_{7}\right\}$, where

$\alpha_{1}=p \wedge \neg q \wedge r \wedge \neg q, \alpha_{2}=p \wedge \neg q \wedge r \wedge \neg q \wedge \neg q, \alpha_{3}=q \wedge \neg p \wedge \neg p \wedge s$, $\alpha_{4}=q \wedge \neg p \wedge \neg p \wedge \neg p \wedge s, \alpha_{5}=(p \leftrightarrow q) \wedge t, \alpha_{6}=(p \leftrightarrow q) \wedge t \wedge t$ and $\alpha_{7}=(p \leftrightarrow q) \wedge t \wedge t \wedge t$. Let $P$ be the profile from Table 21 .

\begin{tabular}{r|cccccccccccccc} 
Voters & $\{p$, & $q$, & $p \wedge q$, & $p \wedge \neg q \wedge r$, & $\alpha_{1}$, & $\alpha_{2}$, & $\neg p \wedge q \wedge s$, & $\alpha_{3}$, & $\alpha_{4}$, & $\alpha_{5}$, & $\alpha_{6}$, & $\left.\alpha_{7}\right\}$ \\
\hline$J_{1}$ & + & + & + & - & - & - & - & - & - & + & + & + \\
$J_{2}$ & + & - & - & + & + & + & - & - & - & - & - & - \\
$J_{3}$ & - & + & - & - & - & - & + & + & + & - & - & - \\
\hline$m(P)$ & + & + & - & - & - & - & - & - & - & - & - & -
\end{tabular}

Table 21: A profile $P$ showing that MPC inc MC.

We obtain

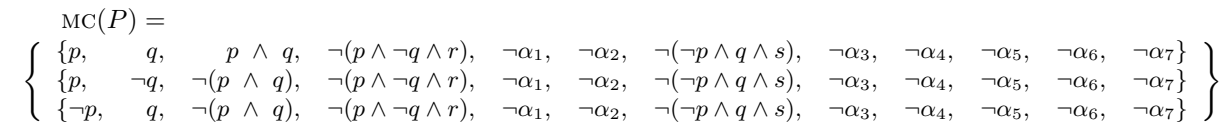

\begin{tabular}{r|ccccccccccccc} 
Voters & $\{p$, & $q$, & $p \wedge q$, & $p \wedge \neg q \wedge r$, & $\alpha_{1}$, & $\alpha_{2}$, & $\neg p \wedge q \wedge s$, & $\alpha_{3}$, & $\alpha_{4}$, & $\alpha_{5}$, & $\alpha_{6}$, & $\left.\alpha_{7}\right\}$ \\
\hline$J_{1}$ & - & - & - & - & - & - & - & - & - & + & + & + \\
$J_{2}$ & + & - & - & + & + & + & - & - & - & - & - & - \\
$J_{3}$ & - & + & - & - & - & - & + & + & + & - & - & - \\
\hline$m(Q)$ & - & - & - & - & - & - & - & - & - & - & - & -
\end{tabular}

Table 22: A profile $Q$ at a minimal $D_{H}$ sitance from $P$ in Table 21. Colouered are the cells with the judgments reversed from $P$

To obtain $\operatorname{MPC}(P)$, we need to change the first three judgments of the first voter, obtaining the profile $Q$ given in Table 22. This is the minimal change, since if either the second or the third agent change either their judgment on $p$ or their judgment on $q$, they have to change additional other three judgments. We obtain $\operatorname{MPC}(P)=\{\{\neg p, \neg q, \neg(p \wedge q), \neg(p \wedge \neg q \wedge$ $\left.\left.r), \neg \alpha_{1}, \neg \alpha_{2}, \neg(p \wedge q \wedge s), \neg \alpha_{3}, \neg \alpha_{4}, \neg \alpha_{5}, \neg \alpha_{6}, \neg \alpha_{7}\right\}\right\}$. Thus, MC inc MPC.

7. MPC is incomparable with MCC: Consider the profile $P$ from Table 23 We have $\operatorname{MPC}(P)=\{\{\neg(p \wedge r), \neg(p \wedge s), q, \neg(p \wedge q)\}\}$ and $\operatorname{MCC}(P)=$ $\{\{p \wedge r, p \wedge s, \neg q, \neg(p \wedge q)\},\{p \wedge r, p \wedge s, q, p \wedge q\}\}$; thus $\operatorname{MPC}(P) \cap \operatorname{MCC}(P)=\emptyset$.

8. MPC inc MED: Consider the pre-agenda $[\mathcal{A}]=\left\{p, q, p \wedge q, p \wedge \neg q, \alpha_{1}, \alpha_{2}, q \wedge\right.$ $\left.\neg p, \alpha_{3}, \alpha_{4}\right\}$, where $\alpha_{1}=p \wedge \neg q \wedge \neg q, \alpha_{2}=p \wedge \neg q \wedge \neg q \wedge \neg q, \alpha_{3}=q \wedge \neg p \wedge \neg p$ and $\alpha_{4}=q \wedge \neg p \wedge \neg p \wedge \neg p$. 


\begin{tabular}{r|cccc} 
Voters & $p \wedge r$ & $p \wedge s$ & $q$ & $p \wedge q$ \\
\hline$J_{1}-J_{6}$ & + & + & + & + \\
$J_{7}-J_{12}$ & + & + & - & - \\
$J_{13}-J_{20}$ & - & - & + & - \\
$J_{21}, J_{22}$ & - & - & - & - \\
\hline$m(P)$ & + & + & + & -
\end{tabular}

Table 23: A profile showing that MPC does not satisfy homogeneity.

\begin{tabular}{c|ccccccccccccc} 
Voters & $\{p$, & $q$, & $p$ & $\wedge$ & $p$ & $\wedge$ & $\wedge q$, & $\alpha_{1}$, & $\alpha_{2}$, & $q \wedge \neg p$, & $\alpha_{3}$, & $\left.\alpha_{4}\right\}$ \\
\hline$J_{1}$ & + & + & + & - & - & - & - & - & - \\
$J_{2}$ & + & - & - & & + & + & + & - & - & - \\
$J_{3}$ & - & + & - & & - & - & - & + & + & + \\
\hline \hline Rules & $\{p$, & $q$, & $p$ & $\wedge$ & $q$, & $p$ & $\wedge$ & $\neg q$, & $\alpha_{1}$, & $\alpha_{2}$, & $q \wedge \neg p$, & $\alpha_{3}$, & $\left.\alpha_{4}\right\}$ \\
\hline MPC & - & - & - & & - & - & - & - & - & - \\
\hline MED & + & + & + & - & - & - & - & - & -
\end{tabular}

Table 24: A profile showing that MPC inc MED.

We obtain $\operatorname{MPC}(P)$ by changing the first three judgments of the first voter. This is the minimal change, since if either the second or the third agent change either their judgment on $p$ or their judgment on $q$, they have to change additional other three judgments. Observe that $\operatorname{MED}(P)=$ $\left\{\left\{p, q, p \wedge q, \neg(p \wedge \neg q), \neg \alpha_{1}, \neg \alpha_{2}, \neg(q \wedge \neg p), \neg \alpha_{3}, \neg \alpha_{4}\right\}\right\}$ since for this judgment set the weight is 17 , and for the remaining three other possible judgment sets the weights are: 14 for the set of the judgment sets of the second, and third voter and 16 for the judgment set $\left\{\neg p, \neg q, \neg(p \wedge q), \neg(p \wedge \neg q), \neg \alpha_{1}, \neg \alpha_{2}, \neg(q \wedge \neg p), \neg \alpha_{3}, \neg \alpha_{4}\right\}$.

Thus MPC inc MED.

9. $F^{d_{H}, \mathrm{MAx}}, F_{\mathrm{rev}}$ and $F^{d_{G}, \Sigma}$ are pairwise incomparable: We give one counterexample for all three pairs. Let $\mathcal{A}_{C}$ be the preference agenda for the set of alternatives $C=\left\{c_{1}, c_{2}, c_{3}, c_{4}\right\}$, together with the transitivity constraint. Consider the profile given in Table 25. The collective judgments obtained by $F_{\mathrm{rev}}, F^{d_{G}, \Sigma}$, and $F^{d_{H}, \mathrm{MAx}}$ are represented in the last five rows of this table. 


\begin{tabular}{r|cccccc} 
Voters & $c_{1} P c_{2}$ & $c_{1} P c_{3}$ & $c_{1} P c_{4}$ & $c_{2} P c_{3}$ & $c_{2} P c_{4}$ & $c_{3} P c_{4}$ \\
\hline$J_{1}$ & + & + & + & - & - & - \\
$J_{2}, J_{3}$ & - & + & + & + & + & + \\
\hline Rule & $c_{1} P c_{2}$ & $c_{1} P c_{3}$ & $c_{1} P c_{4}$ & $c_{2} P c_{3}$ & $c_{2} P c_{4}$ & $c_{3} P c_{4}$ \\
\hline \hline$F_{\text {rev }}\left(\left\langle J_{1}, J_{2}, J_{3}\right\rangle\right)$ & + & + & + & + & + & + \\
$F_{G}^{d_{G}, \Sigma}\left(\left\langle J_{1}, J_{2}, J_{3}\right\rangle\right)$ & - & + & + & + & + & + \\
$F^{d_{H}, \operatorname{Max}}\left(\left\langle J_{1}, J_{2}, J_{3}\right\rangle\right)$ & + & + & + & - & + & + \\
& + & + & + & + & - & + \\
& + & + & + & + & + & -
\end{tabular}

Table 25: A profile showing that $F^{d_{H}, \mathrm{MAx}}, F_{\mathrm{rev}}$ and $F^{d_{G}, \Sigma}$ are mutually incomparable. 